Key Words:

XAFS

Zeolites

Sodalite

Uranium

Retention:

Permanent

\title{
Characterization of Uranium Solids Precipitated with Aluminosilicates
}

\author{
Martine C. Duff, Douglas B. Hunter and Lawrence N. Oji
}

REPORT DATE NOVEMBER 26, 2002

Westinghouse Savannah River Company

Savannah River Site

Aiken, SC 29808

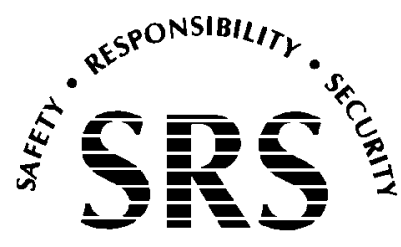

SAVANNAH RIVER SITE

Prepared for the U.S. Department of Energy Under

Contract Number DE-AC09-96SR18500 
This document was prepared in conjunction with work accomplished under Contract No. DE-AC09-96SR18500 with the U. S. Department of Energy.

\section{DISCLAIMER}

This report was prepared as an account of work sponsored by an agency of the United States Government. Neither the United States Government nor any agency thereof, nor any of their employees, makes any warranty, express or implied, or assumes any legal liability or responsibility for the accuracy, completeness, or usefulness of any information, apparatus, product or process disclosed, or represents that its use would not infringe privately owned rights. Reference herein to any specific commercial product, process or service by trade name, trademark, manufacturer, or otherwise does not necessarily constitute or imply its endorsement, recommendation, or favoring by the United States Government or any agency thereof. The views and opinions of authors expressed herein do not necessarily state or reflect those of the United States Government or any agency thereof.

This report has been reproduced directly from the best available copy.

Available for sale to the public, in paper, from: U.S. Department of Commerce, National Technical Information Service, 5285 Port Royal Road, Springfield, VA 22161, phone: (800) 553-6847, fax: (703) 605-6900

email: orders@ntis.fedworld.gov

online ordering: http://www.ntis.gov/help/index.asp

Available electronically at http://www.osti.gov/bridge

Available for a processing fee to U.S. Department of Energy and its contractors, in paper, from: U.S. Department of Energy, Office of Scientific and Technical Information, P.O. Box 62, Oak Ridge, TN 37831-0062,

phone: (865)576-8401,

fax: (865)576-5728

email: $\underline{\text { reports@ adonis.osti.gov }}$ 
Key Words:

XAFS

Zeolites

Sodalite

Uranium

Retention:

Permanent

\title{
Characterization of Uranium Solids Precipitated with Aluminosilicates
}

\author{
Martine C. Duff, Douglas B. Hunter and Lawrence N. Oji
}

REPORT DATE NOVEMBER 26, 2002

Westinghouse Savannah River Company

Savannah River Site

Aiken, SC 29808

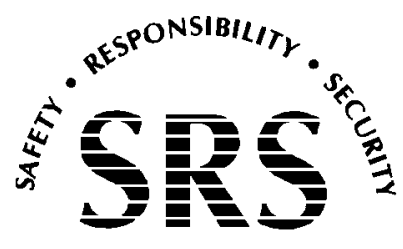

SAVANNAH RIVER SITE

Prepared for the U.S. Department of Eneingy Under

Contract Number DE-AC09-96SR18500 


\section{REVIEWS AND APPROVALS}

M. C. Duff, Author, Waste Processing Technology

Date

D. B. Hunter, Author, Non-Proliferation Technology

Date

L. N. Oji, Author, Waste Processing Technology

Date

B. Van-Pelt, Level 4 Manager, Waste Processing Technology

Date

R. E. Edwards, Manager, Process Engineering

Date

W. L. Tamosaitis, Manager, Waste Processing Technology

Date

D. T. Hobbs, Design Check, Waste Processing Technology

Date 


\section{TABLE OF CONTENTS}

LIST OF FIGURES 2

LIST OF TABLES 3

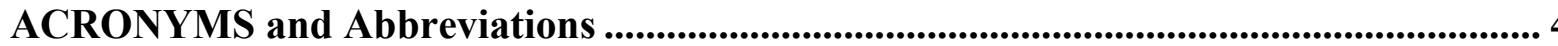

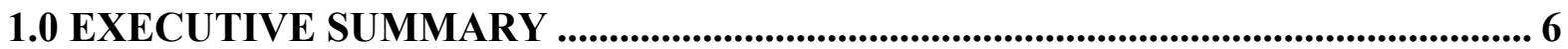

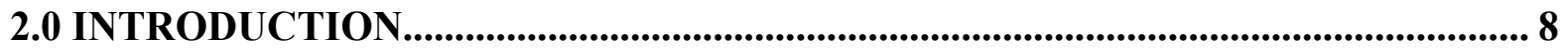

2.1 Potential Routes of U Accumulation with NAS ..................................................................... 8

2.2 Review of Uptake Studies with U(VI) and Zeolites .............................................................. 12

2.3 Use of XAFS Techniques to Characterize Metal Uptake by Surfaces........................... 13

2.4 Experimental Objectives............................................................................................... 13

3.0 MATERIALS AND EXPERIMENTAL METHODS.................................................... 14

3.1 Sample Preparation .................................................................................................................. 14

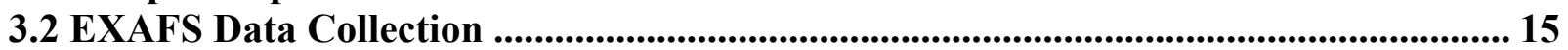

3.3 XANES and EXAFS Data Analyses........................................................................... 16

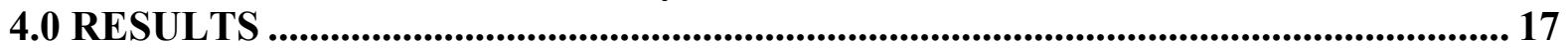

4.1 Background on the Characterization of $U$ Behavior on Surfaces with XAFS.............. 17

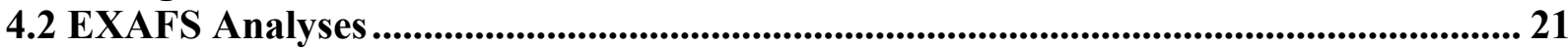

4.2.1 Uranium Chi Data for U(VI)-Loaded NAS U Reference Samples .......................... 21 4.2.1.1 Amorphous Zeolite Precursor Samples and the Si-free Reference Sample for the Amorphous Zeolite Syntheses ........................................................................................ 21 4.2.1.2 Sodalite Samples and the Si-free Reference Sample for the Sodalite Syntheses

4.2.1.3 Si-free Reference Sample for the Zeolite A Syntheses............................................ 21

4.2.2 Uranium Fourier-Transformed Data ............................................................................... 25

4.2.2.1 Fourier-Transformed Data for $U$ in the U(VI)-containing Amorphous Zeolite and Corresponding Si-free U Reference Samples............................................................... 25

4.2.2.2 Fourier-Transformed Data for $U$ in the U(VI)-containing Sodalite and Corresponding Si-free U Reference Samples ........................................................................ 25

4.2.3 Uranium FT and Model Fit Data ................................................................................. 29

4.2.3.1 Fourier-Transformed Data for $U$ in the Si-free $U$ Reference Sample for the Amorphous Zeolite Synthesis ............................................................................................. 29 4.2.3.2 Fourier-Transformed Data for $U$ in the Si-free $U$ Reference Sample for the Sodalite Synthesis...................................................................................................................... 29 4.2.3.3 Fourier-Transformed Data for $U$ in the Si-free $U$ Reference Sample for the Zeolite A Synthesis............................................................................................................... 34 4.2.3.4 Fourier-Transformed Data for $U$ in Carbonate-washed Amorphous Zeolite. 37 4.2.3.5 Fourier-Transformed Data for $U$ in Carbonate-washed Amorphous Sodalite37 4.2.3.6 Influence of Multiple Local Coordination Environments on the U-XAFS Data Analyses ............................................................................................................................ 41

5.0 DISCUSSION AND CONCLUSIONS .......................................................................... 42

5.1 Discussion of the XAFS Data from the Si-free U Reference Solids................................. 42

5.2 Discussion of the XAFS Data from the Unwashed, DI-washed and Carbonate-washed

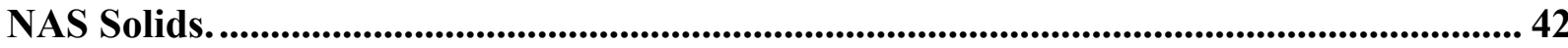

6.0 QUALITY ASSURANCE ......................................................................................... 44

7.0 ACKNOWLEDGEMENTS ................................................................................................ 44

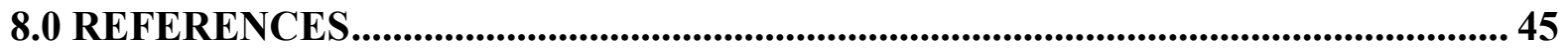




\section{LIST OF FIGURES}

Figure 2.1 Simplified diagram of the types of associations a metal could have with a surface: A) Structural incorporation/co-precipitation, B) Outer-sphere (electrostatic) sorption, C) Specific or inner-sphere sorption and D) Surface precipitation. Blue rings denote first, second and third shell environments that can be probed with X-ray absorption fine-structure

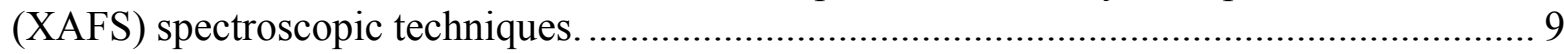

Figure 2.2 Pictorial representation of possible U interactions with NAS and related solids........ 11

Figure 3.1 Diagram of the downstream portion of the hutch table at NIST beamline X23a2 (not drawn to scale). 16

Figure 4.1 Pictorial representation of U(VI) edge and corner sharing with silica tetrahedra....... 18

Figure 4.2 Examples of uranyl silicate structures. Red arrows denote radial U-U distances that can be obtained using crystallographic and XAFS analyses............................................. 20

Figure 4.3 The $k^{3}$-weighted chi data (the plot of the wavevector in reciprocal space) for the amorphous zeolite samples and Si-free $\mathrm{U}$ reference samples. ............................................ 22

Figure 4.4 The $k^{3}$-weighted chi data (the plot of the wavevector in reciprocal space) for the sodalite samples and the Si-free U reference sample.

Figure 4.5 The $k^{3}$-weighted chi data (the plot of the wavevector in reciprocal space) for the $\mathrm{Si}$ free zeolite A U reference sample.............................................................................. 24

Figure 4.6 FT data for the amorphous zeolite series samples - uncorrected for phase shift........ 26 Figure 4.7 The two types of U(VI) first shell bonding environments.' The solids with uranate in their names such as sodium diuranate consist of uranyl-type bonding. Hence, the name of the solid does not always describe the nature of the bonding.....

Figure 4.8 FT data for the sodalite series samples - uncorrected for phase shift........................ 28

Figure 4.9 FT and first coordination shell fit data for the Si-free $\mathrm{U}$ reference (for the amorphous zeolite synthesis) — uncorrected for phase shift. .................................................................... 31

Figure 4.10 FT and first coordination shell fit data for the Si-free U reference sample (for the sodalite synthesis) - uncorrected for phase shift.................................................................. 32

Figure 4.11 FT and second coordination shell fit data for the U reference sample (for the sodalite synthesis) - uncorrected for phase shift.] Although no Si was added to this sample, the best

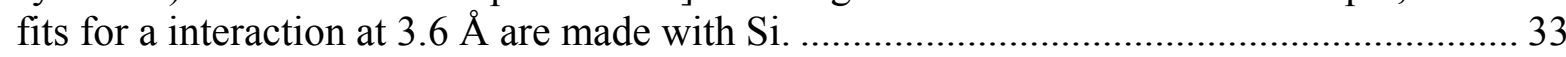

Figure 4.12 FT and first shell fit data for the U reference sample (for the zeolite A synthesis) -

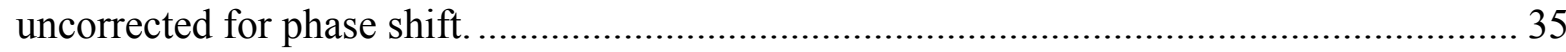

Figure 4.13 FT and outer shell fit data for the U reference sample (for the zeolite A synthesis) -

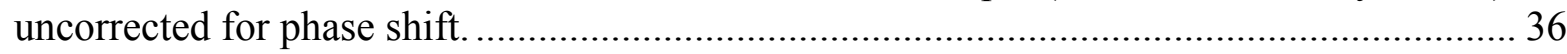

Figure 4.14 FT and multiple shell fit data for the carbonate-washed amorphous zeolite sample-

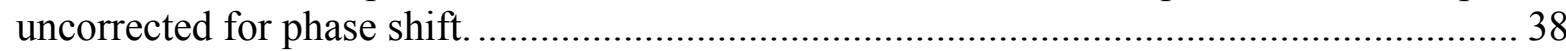

Figure 4.15 FT and first shell fit data for the carbonate-washed sodalite sample- uncorrected for phase shift. 39

Figure 4.16 FT and outer shell fit data for the carbonate-washed sodalite sample- uncorrected for phase shift. 40 
WSRC-TR-2002-00510, REVISION 0

\section{LIST OF TABLES}

Table 3-1 The U-loaded NAS samples that were analyzed with XAFS based on ICP-MS determinations from Oji and Williams (2002).

Table 4-1 Literature XAFS fit data for sorbed U(VI) on characterized Al and Si minerals. The magnitude of the Debye-Waller Factor $\left[\sigma^{2}[\AA]^{2}\right]$ indicates the variation in the bond length

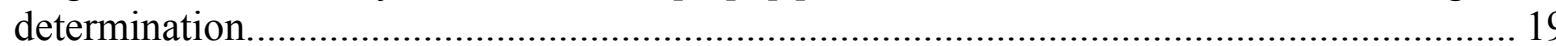




\section{ACRONYMS AND ABBREVIATIONS}

Al

$\mathrm{Ca}$

$\mathrm{CO}_{3}{ }^{2-}$

$\mathrm{CN}$

$\delta(\mathrm{K})$

DI

DWPF

EXAFS

E

$\mathrm{E}_{0}$

$\mathrm{E}_{0}$ Shift

$\mathrm{F}$

$\mathrm{Fe}$

FEFF

FFIT

FT

$\hbar$

HLW

$k$

$\mathrm{m}$

MST

$\mathrm{Na}$

NAS

$\mathrm{Na}_{8}\left(\mathrm{AlSiO}_{4}\right)_{6} \cdot \mathrm{nH}_{2} \mathrm{O}_{(\mathrm{s})}$

$\mathrm{Na}_{8}\left(\mathrm{AlSiO}_{4}\right)_{6} \cdot \mathrm{nH}_{2} \mathrm{O}_{(\mathrm{s})}$

$\mathrm{Na}_{6}\left(\mathrm{AlSiO}_{4}\right)_{6} \cdot \mathrm{nH}_{2} \mathrm{O}_{(\mathrm{s})}$

$\mathrm{Na}_{12} \mathrm{Al}_{12} \mathrm{Si}_{12} \mathrm{O}_{48} \cdot 27 \mathrm{H}_{2} \mathrm{O}_{(\mathrm{s})}$

NSLS

$\mathrm{O}$

R-space

$\mathrm{RDF}$

RT

$\mathrm{S}^{\wedge} 2$ or $\mathrm{S}_{0}^{2}$

$\sigma^{2}$ or $\operatorname{SIGMA}^{2}$

Si

$\mathrm{Si}(\mathrm{Li})$

$\mathrm{Sr}$
Aluminum

Calcium

Carbonate ion

Coordination number

Electronic phase shifts due to atomic potentials

De-ionized water

Defense Waste Processing Facility

Extended X-ray Absorption Fine-Structure

$E$ is the kinetic energy of the photoelectron

EXAFS defined edge energy in electron volts or $\mathrm{eV}$ (not equal to edge energy as defined by XANES but is equal to the energy of the photoelectron at $k=0$.

A relative value of $E_{0}$ (a variable in the EXAFS Equation)

Backscattering amplitude of the atom

Iron

An automated computer program for making ab initio multiple scattering calculations of XAFS and XANES spectra for atoms.

A Levenberg-Marquardt fitting program created by researchers at the University of Washington

Fourier-transform

Plank's constant

High Level Waste

Chi where $k$ is the square root of $\left[\left(2 \mathrm{~m} / \hbar^{2}\right) *\left(E-E_{0}\right)\right]$

Mass of the photoelectron

Monosodium titanate

Sodium

Sodium Aluminosilicates

Sodalite (generic form)

Cancrinite (generic form)

Zeolite A (generic form)

Amorphous zeolite precursor material

National Synchrotron Light Source

Oxygen

$\mathrm{R}$-space pertains to mean atom position or bond distance (radial distance in $\AA$ )

Radial Distribution Function

Room Temperature

Amplitude reduction factor (EXAFS Equation variable associated with central atom shake-up and shake-off effects)

Debye-Waller Factor or Relative Mean Square Disorder in bond length (a variable in the EXAFS Equation)

Silica (or silicon)

An energy dispersive Li-doped silicon detector

Strontium 
SRTC

$\mathrm{U}$

$\left(\mathrm{UO}_{2}\right)_{2} \mathrm{SiO}_{4} \cdot 2 \mathrm{H}_{2} \mathrm{O}$

$\mathrm{U}(\mathrm{VI})$

$\mathrm{U}(\mathrm{VI}) \mathrm{O}_{3} \cdot 2 \mathrm{H}_{2} \mathrm{O}_{(\mathrm{s})}$

WSRC

XAFS

XANES

XRD
Savannah River Technology Center

Uranium

Soddyite

Hexavalent uranium

Schoepite, a uranyl oxyhydroxide mineral

Westinghouse Savannah River Company

X-ray Absorption Fine-Structure (XANES plus EXAFS)

$\mathrm{X}$-ray Absorption Near-Edge Structure

$\mathrm{X}$-ray Diffraction 
WSRC-TR-2002-00510, REVISION 0

\subsection{EXECUTIVE SUMMARY}

At the Savannah River Site (SRS), the High-Level Waste (HLW) Tank Farms store and process high-level liquid radioactive wastes from the Canyons and recycle water from the Defense Waste Processing Facility (DWPF). The waste is concentrated using evaporators to minimize the volume of space required for HLW storage. After evaporation, the waste concentrate is transferred to one or more receipt storage tanks. Recently, the $2 \mathrm{H}$ Evaporator was shutdown due to crystallization of sodium aluminosilicates (NAS) (such as cancrinite, sodalite, zeolite A and various zeolite precursor materials) that contained elementally enriched uranium (U) solids. The evaporator deposits resided on the evaporator walls and other exposed internal surfaces within the evaporator pot.

Little is known about the interaction between U and NAS in HLW salt solutions. This research was conducted to improve our fundamental understanding of the mechanisms of $U$ accumulation with NAS in the evaporators and in other process areas at the SRS that may concentrate $U$ in the presence of silicates, aluminum and NAS. These fundamental studies will help support the basis for the continued safe operation of SRS evaporators and this fundamental information will be used to help mitigate $U$ accumulation during evaporator operation.

To examine the interaction of U with NAS solids, Savannah River Technology Center (SRTC) conducted tests to examine the local structural speciation of $U$ following NAS phases: amorphous zeolite precursor material, sodalite $\left[\mathrm{Na}_{8}\left(\mathrm{AlSiO}_{4}\right)_{6} \cdot \mathrm{nH}_{2} \mathrm{O}_{(\mathrm{s})}\right]$ and zeolite $\mathrm{A}$ $\left[\mathrm{Na}_{12} \mathrm{Al}_{12} \mathrm{Si}_{12} \mathrm{O}_{48} \cdot 27 \mathrm{H}_{2} \mathrm{O}_{(\mathrm{s})}\right]$. The experiments were designed to obtain simultaneous formation of NAS and U solids. The solids were analyzed using X-ray absorption fine-structure (XAFS) spectroscopic techniques. The U-containing NAS solids were also analyzed after washing with deionized (DI) water and with carbonate-containing solutions. The studies yielded the following information:

- In general, the U(VI) that was removed from solution during these studies precipitated as two general forms: mainly U(VI)-oxide/hydroxide phases with minor U(VI)-silicates phases. The $\mathrm{U}(\mathrm{VI})$-silicate phases probably formed due to the high dissolved Si levels that were required for NAS precipitation. Although the U(VI)-silicates were the minor phases, they were the most stable phases because of their resistance to dissolution in the leaching solutions.

- The unwashed and DI-washed U-NAS precipitates that form in the presence of amorphous zeolite precursor material and sodalite possess multiple local coordination environments for the U. These environments consist of uranyl oxyhydroxide and uranyl silicate precipitate phases, which probably have a strong amorphous character.

- For the DI- and carbonate-washed samples, the average local environment of the U becomes less characteristic of multiple environments and more characteristic of an amorphous colloidal uranyl silicate phase than of a uranyl oxyhydroxide phase. This uranyl silicate material has bonding characteristics of uranyl silicate minerals but no identifications to known U(VI)-silicate minerals could be made. Follow-up studies to determine the solubility of this phase will be complicated due to the colloidal nature of the uranyl silicate materials. 
- For these DI-water and carbonate washed samples, the presence of silicon ( $\mathrm{Si}$ ) at distances that are representative of corner sharing [of silicate tetrahedra $\left(\mathrm{SiO}_{4}\right)$ with $\mathrm{U}(\mathrm{VI})$ polyhedra] indicate that U(VI) exists in a solid phase precipitate form with the NAS as opposed to sorbed on the NAS. We cannot determine whether there is a seeding relationship between the U and NAS solids from our data or whether these solids did not dissolve in the wash solutions due to their occlusion by NAS solids.

To determine when the U should be added during these NAS syntheses procedures, precipitation timing studies were done with $\mathrm{U}, \mathrm{Al}$ and caustic salt solutions using the same experimental conditions (such as temperature) as those required for the individual NAS syntheses. (No Si was added to avoid making NAS for these reference $U$ materials.) Our study acquired data for the $U$ reference solids and the studies yielded the following information:

- The U reference solids that were prepared using the same methods as those for amorphous zeolite precursor material, zeolite A and sodalite (i.e., with Al and U(VI), but without Si) were analyzed with XAFS. Radial distance and atom identity information for the $U$ in these samples indicate that first coordination shell bonding was to oxygen atoms, which is typical for U(VI) species. The uranium-XAFS data for the precipitated solids from the $U$ reference for the sodalite and zeolite A synthesis materials indicate that bonding with $\mathrm{Al}$ (in the outer coordination shells) may have occurred. It is also possible that bonding with "tramp" $\mathrm{Si}$ (which may have come from the reagent chemicals used to make the salt solutions) occurred instead of bonding with Al. The XAFS data for the Si-free U reference solid from the amorphous zeolite precursor preparation did not indicate the presence of Al bonding in the outer coordination shells.

Further study is recommended to evaluate the potential for the formation of U-Al solid phases in the absence of added Si. Additionally, a study of the influence of Si on U(VI) solubility under alkaline conditions with temperature is recommended because little information exists about $U$ under these conditions. Finally, if tank farm waste containing elevated levels of Pu were to be evaporated, studies on the formation and characterization of Pu silicate phases would also be recommended. 
WSRC-TR-2002-00510, REVISION 0

\subsection{INTRODU CTION}

\subsection{Potential Routes of U Accumulation with NAS}

Uranium accumulation during the evaporation of HLW is a potential criticality risk if the incoming waste is enriched in ${ }^{235} \mathrm{U}$. During evaporation, several processes may contribute to the accumulation of U-containing solids as shown pictorially in Fig. 2.1 and 2.2. [Note: Fig. 2.1 demonstrates potential U interactions with NAS on the molecular scale whereas Fig. 2.2 shows more of a conceptual or cartoon-like diagram of potential U interactions with NAS solids.] This report will operationally define the various uptake mechanisms according to the following text descriptions.

Uptake processes by solids can occur by several mechanisms: structural incorporation, ion exchange (electrostatic or outer-sphere) sorption, specific adsorption and surface precipitation. Fig. 2.1 provides a schematic of these processes. Many of these terms have been used loosely in the literature and their use varies with scientific discipline. This report focuses on NAS surfaces and their interaction with U. A more thorough review of these processes that that presented in the following paragraphs is presented elsewhere. ${ }^{1,2}$ The expression of these uptake processes typically depends upon the amount of metal added, solution and solid phase characteristics.

"Ion exchange" can have different meanings, which results in some confusion in the literature. One very general way to express ion exchange is Structural Incorporation, which is the substitution of a solution species for a structural atom as shown in the example below for strontium $(\mathrm{Sr})$ ion substitution for calcium $(\mathrm{Ca})$ ion in calcite [calcium carbonate, $\mathrm{CaCO}_{3(\mathrm{~s})}$ ]

$$
\mathrm{CaCO}_{3(\mathrm{~s})}+\mathrm{Sr}^{2+} \longrightarrow \mathrm{SrCO}_{3(\mathrm{~s})}+\mathrm{Ca}^{2+}
$$

This type of metal uptake typically requires ready access to structural atoms. One example of this process would be the exchange of $\mathrm{Na}^{+}$for $\mathrm{Ca}^{2+}$ within the interlayer region of a layered metal oxide solid. Structural incorporation also involves co-precipitation, where a new atom substitutes for one or more of the host atoms in a structure. Ideally, structural incorporation of anions could also result in a similar number of neighboring atoms in the first coordination shell to that of the replaced ion prior to its release. Therefore, the local environment of the exchanged atom would tend to resemble the environment the newly exchanged atom had before its release (Fig. 2.1A). Two exceptions to this would be when the host structure is a nanoparticle and when the structure is amorphous. In these cases, exact determinations of whether the participating metal species resides in a structural vacancy or within an interlayer site are not straightforward. ${ }^{3}$ 


\section{Molecular/Atom Scale Metal Interactions with Surfaces}

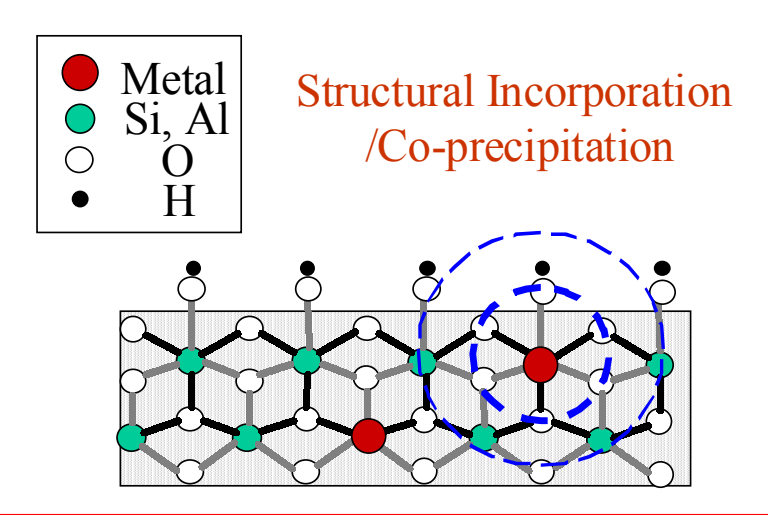

Specific Adsorption/ Chemisorption / Inner Sphere Sorption

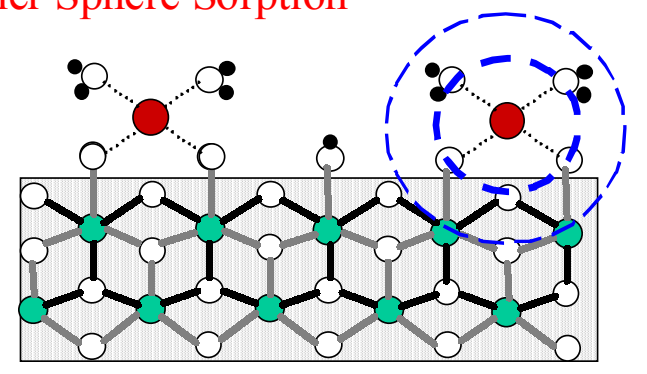

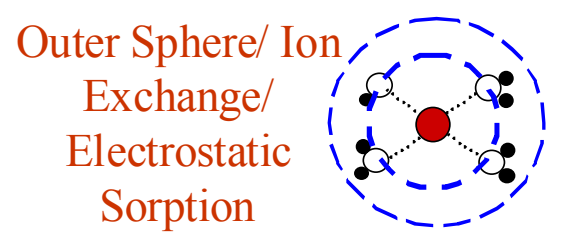

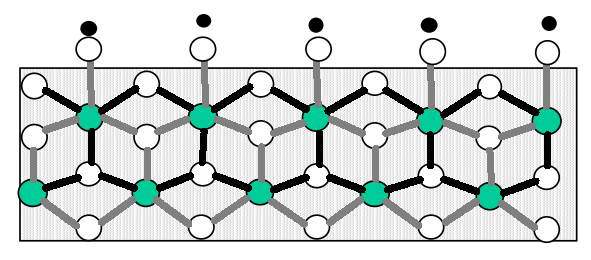

\section{Surface Precipitation/Polymerization}

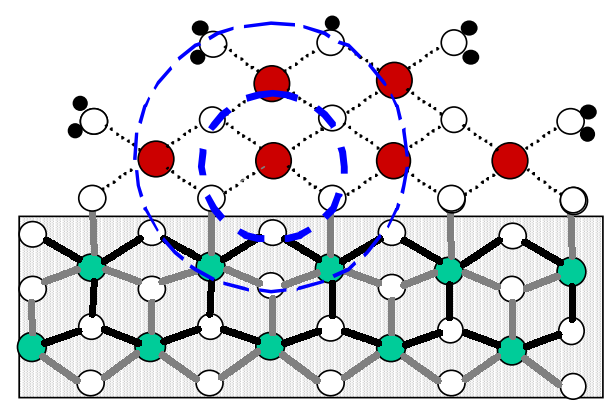

Blue circles denote coor dination shells.

Figure 2.1 Simplified diagram of the types of associations a metal could have with a surface: A) Structural incorporation/co-precipitation, B) Outer-sphere (electrostatic) sorption, C) Specific or inner-sphere sorption and D) Surface precipitation. Blue rings denote first, second and third shell environments that can be probed with X-ray absorption fine-structure (XAFS) spectroscopic techniques. 
Ion Exchange in a more restrictive sense as used in this report is an electrostatic process involving the replacement of one readily exchangeable hydrated ion by another similarly exchangeable ion (Fig. 2.1B). This type of sorption is also referred to as Outer Sphere sorption. Ion exchange does not involve the formation of bonds on the participating surface because the sorbed ion is only present in the diffuse double layer (DDL). This type of outer sphere sorption is normally reversible ${ }^{4}$ and is a function of ionic strength (i.e., such as $\mathrm{Na}^{+}$ion concentration). At high ionic strengths, the DDL collapses and less sorption by ion exchange is observed than at low ionic strengths. Ion exchange sorption is often associated with materials that have constant surface charge and exhibit no change in overall surface charge upon ion exchange sorption. An example of this process would be exchange of hydrated $\mathrm{Na}^{+}$ion for hydrated $\mathrm{UO}_{2}{ }^{2+}$ in the DDL.

Specific adsorption involves the formation of predominantly covalent bonds with the surface, but the bonds can have some ionic behavior. This type of sorption is often referred to as Chemisorption or Inner Sphere sorption. Specifically adsorbed metals typically have one or more atoms from the participating surface in the second coordination shell (Fig. 2.1C). This type of sorption involves the release of $\mathrm{H}^{+}$or structural surface ions (such as $\mathrm{Na}^{+}$in monosodium titanate or MST) upon sorption. For example, specific sorption of $\mathrm{UO}_{2}{ }^{2+}$ to NAS could result in the presence of $\mathrm{Si}$ and $\mathrm{Al}$ atoms in the second coordination shell of the $\mathrm{UO}_{2}{ }^{2+}$. Specific adsorption is usually irreversible. ${ }^{4}$ However, in the literature, specific adsorption is not always differentiated from structural incorporation or surface precipitation. Specific adsorption may involve mononuclear complexes or polymeric species. It may occur with metals and their associated ligands [such as a U(VI)-carbonate ion] and it is influenced by other solution- and surface-related variables. ${ }^{5}$

Surface Precipitation occurs by nucleation of new solid phase on a host surface (Fig. 2.1D). For example, when the concentration of a dissolved metal such as $\mathrm{UO}_{2}{ }^{2+}$ is high enough to result in the super-saturation of one or more $\mathrm{UO}_{2}{ }^{2+}$-containing phases [such as schoepite, which has a formula of $\mathrm{U}(\mathrm{VI}) \mathrm{O}_{3} \cdot 2 \mathrm{H}_{2} \mathrm{O}_{(\mathrm{s})}$ ] in the presence of another solid (such as NAS), the other solid facilitates the nucleation of the new solid $\mathrm{UO}_{2}{ }^{2+}$-rich phase. This U-rich material would have numerous $U$ atoms in the second coordination shell of the $U$. The formation of colloidal (polymeric) U species on surfaces would resemble the same local environment (on the atom scale) as observed for surface precipitation. When atoms from a potential host surface are absent and polymeric species are present, the mechanism of $U$ uptake from solution is likely to be direct homogeneous (solid phase) precipitation.

Uranium may be concentrated by sorption to the surfaces of the NAS, precipitation within NAS structures and precipitation as U phases. These processes are illustrated in a more simplistic manner in Fig. 2.2, which emphasizes the potential interactions U can have with NAS. Sorption can be divided into two types of molecular scale sorption-related processes and it involves the uptake of atoms near or at a participating sorptive surface (Fig. 2.2A). An element such as $U$ could co-precipitate with the NAS and related solids as shown pictorially in Fig. 2.2B. [For zeolites, the term co-precipitation could be sub-divided to include uptake into zeolite channels and any isomorphic substitution (i.e., of $\mathrm{U}$ for $\mathrm{Si}$ or $\mathrm{Al}$ ) in the zeolite structure. ${ }^{6}$ ] Uranium could 
A) Sorption of U on NAS (may facilitate nucleation of U solid phases)

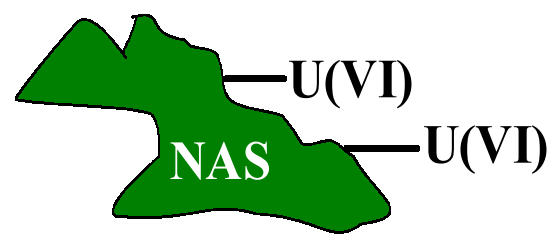

B) Co-precipitation of $U$ within crystalline or amorphous NAS structures

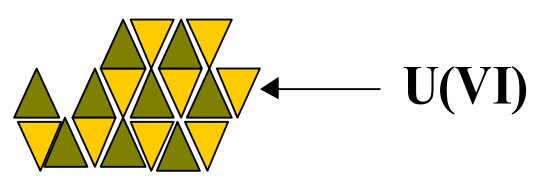

D) Precipitation of $U$ as a U(VI) oxide, oxide hydrate, nitrate, or silicate

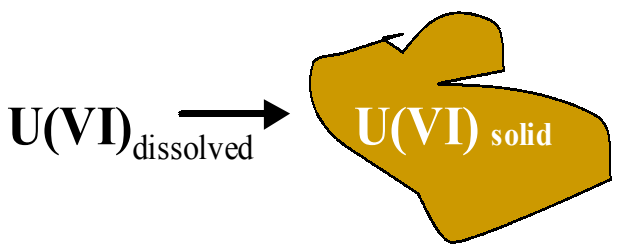

E) Silica sols facilitate greater U pseudosolubility (possible desorption of $U$ from other phases upon silica addition?)

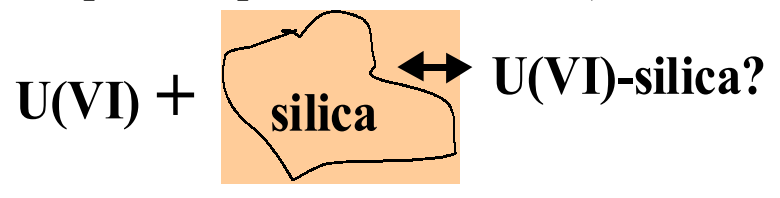

C) Occlusion: growth of $U$ solids on precipitating NAS solids and growth of NAS solids on precipitating $\mathrm{U}$ solids

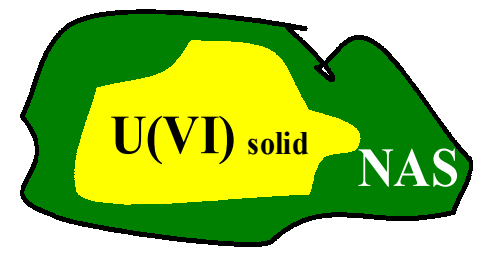

Figure 2.2 Pictorial representation of possible U interactions with NAS and related solids. 
also deposit by precipitation via surface nucleation (often referred to as chemical seeding) on NAS minerals (Fig. 2.2C). It is also possible that U solids could seed the growth of NAS solids (Fig. 2.2C). Uranium could potentially precipitate as an oxide [such as $\mathrm{UO}_{3(\mathrm{~s})}$, a hydrous oxide [such as $\mathrm{Na}_{2}\left(\mathrm{UO}_{2}\right)_{3} \mathrm{O}_{3} \cdot(\mathrm{OH})_{2}$ (s) , and a silicate [e.g., $\left(\mathrm{UO}_{2}\right)_{2} \mathrm{SiO}_{4} \cdot 2 \mathrm{H}_{2} \mathrm{O}_{(\mathrm{s})}$ and $\mathrm{Na}_{4}\left(\mathrm{UO}_{2}\right)_{2} \mathrm{Si}_{4} \mathrm{O}_{10} \cdot$ $4 \mathrm{H}_{2} \mathrm{O}_{(\mathrm{s})}$ ] (Fig. 2.2D). ${ }^{7,8}$ Precipitation of $\mathrm{U}$ could occur simultaneously with the precipitation of NAS solids. This process is referred to as solid phase nucleation. ${ }^{1}$ The precipitation of $U$ could also occur independent of NAS formation. A molecular scale view of an example of a $U$ precipitate is not presented in Fig. 2.2. However, a crystalline $U$ form not associated with another separate mineral surface would be identifiable based on its crystal structure and the positions and identities of the atoms in that structure. This information is obtained from structural refinements of X-ray diffraction (XRD) data and from XAFS data.

Uranium may also interact with silica sols, which have no defined crystal structure because of their amorphous nature (Fig. 2.2E). This interaction could possibly enhance U solubility by forming U-silica colloids, which appear (by analysis) to be dissolved because they are too small to be isolated by solution filtration. At an atom or molecular scale basis, this type of interaction with $U$ is best be described by the description of structural incorporation in Fig. 2.1A, which shows $\mathrm{U}$ in a crystalline structural-type environment. However, for colloidal Si, which is often very amorphous, the local environment of the $U$ would not resemble an environment that has a crystalline structure.

\subsection{Review of Uptake Studies with U(VI) and Zeolites}

Several solid phases or sorbents such as the titanates (such as monosodium titanate or MST) and Mn oxides concentrate actinides and other radionuclides under conditions that are similar to alkaline HLW salt solutions. ${ }^{9}, 10,11,12$ At high $\mathrm{pH}$, the surfaces of most solids are negatively charged and likely to sorb cationic species, such as $\mathrm{Sr}^{2+}$. Under these solution conditions most actinides such as $\mathrm{U}(\mathrm{VI})$ exist as negatively charged hydrolysis and carbonate $\left(\mathrm{CO}_{3}{ }^{2-}\right)$ species [such as $\left(\mathrm{UO}_{2}\right)_{2}(\mathrm{OH})_{5}{ }^{-}$and $\left.\mathrm{UO}_{2}\left(\mathrm{CO}_{3}\right)_{3}{ }^{4-}\right] .{ }^{13}$ When these $\mathrm{U}(\mathrm{VI})$ species dominate the solution speciation (at high $\mathrm{pH}$ or in high $\mathrm{CO}_{3}{ }^{2-}$ solutions), $\mathrm{U}(\mathrm{VI})$ typically has a low affinity for certain solids, such as the Fe oxides. ${ }^{14,15}$ The sorption of U(VI) species among other actinide species on sorbents such as the titanates occurs to a limited extent at high $\mathrm{pH}$. The mechanisms by which these processes occur with titanates, which are likely to be negatively charged are not known although some information exists. ${ }^{16}$ Sorption studies with U(VI) typically demonstrate that it has low affinity for zeolites at high $\mathrm{pH}$ (9-11) — particularly in the presence of elevated inorganic carbon. $^{17,18,19,20}$ This is because the higher levels of dissolved inorganic carbon favor the formation of negatively-charged, highly soluble U(VI)-carbonate complexes, which have low affinities for similarly-charged surfaces. Few sorption studies have been done with $U$ and zeolites above $\mathrm{pH}$ 12. Spectroscopic studies with silicate gels indicate that U sorbs via edge sharing with silica tetrahedra. ${ }^{21}$ This type of sorption to surface via edge linking (as opposed to corner linking) is typical for sorbed U(VI) species. ${ }^{22,23}$

Studies that have synthesized zeolites in the presence of U(VI) show that uptake can occur and results in deformities within the zeolite structure. Under acidic conditions, these deformities permit $\mathrm{U}$ uptake at locations that would normally have $\mathrm{Na}^{+}$or $\mathrm{H}^{+}$ions-depending on the form (i.e., $\mathrm{Na}^{+}$or $\mathrm{H}^{+}$) of the zeolite. ${ }^{24}$ Other studies that have examined the uptake of $\mathrm{U}$ from highly 
WSRC-TR-2002-00510, REVISION 0

acidic solutions that are precipitating zeolites indicate that the $\mathrm{U}$ is in dimeric cavities of the zeolites as hydrated forms such as $\left[\mathrm{UO}_{2}(\mathrm{OH})_{4}\right]^{2-25}$

\subsection{Use of XAFS Techniques to Characterize Metal Uptake by Surfaces}

The local environment of metals associated with surfaces can be investigated with analytical techniques such as X-ray absorption fine-structure (XAFS) spectroscopy. We used XAFS to study the local structural environment of $U$ that has precipitated with NAS solids. The XAFS spectroscopic techniques are among the best for providing detailed chemical speciation information in environmental samples - particularly when information from multiple characterization techniques is available. The term XAFS is applicable to both X-ray absorption near-edge structure (XANES) and extended X-ray absorption fine-structure (EXAFS) spectroscopic techniques. The XAFS spectra give robust local structural information on coordination number $(\mathrm{CN})$, bonding symmetry, neighbor and near-neighbor atomic distances and bond disorder (as the root mean square deviations of distances about the average values). Additionally, the information gained is atom specific - making it a versatile technique for structural determinations of atom clusters. ${ }^{26,27}$ XAFS spectroscopy in particular has been successfully applied to the structural elucidation of metal clusters and sorbed metals on surfaces because the technique does not require long range order (i.e., periodicity) or crystalline samples. Hence, this technique can characterize colloidal forms of ions and species that would otherwise be undetected by X-ray diffraction (XRD) because of their poorly crystalline behavior.

\subsection{Experimenta I Objectives}

The primary objective of this research was to obtain information on speciation of $U$ [added as U(VI)] associated with NAS solids that were synthesized with dissolved U using XAFS. Uranium-XAFS analyses were also conducted on solids that had been washed with solutions of DI water. XAFS analyses were also performed after washing with DI water and sodium carbonate $(0.4 \mathrm{M}){ }^{30}$ Washing U-loaded solids with carbonate solutions has been shown to remove sorbed forms of $\mathrm{U}(\mathrm{VI})$, in addition to dissolving the readily soluble (i.e., rapidly dissolving) solid phase forms of $U(V I) .^{22}$ This is due to the formation of highly soluble uranyl carbonate complexes, such as the uranyl di and tri-carbonato species, which typically have low affinities for sorbents, such as the aluminosilicates ${ }^{28}$ and metal oxides. ${ }^{15}$ We used XAFS techniques to obtain information on the average local structural speciation of the $\mathrm{U}$ such as $\mathrm{CN}$, geometry, near and next nearest neighbor environment of the target metal. To determine whether our results were realistic, we used molecular models to evaluate the findings from our EXAFS studies.

This work is part of a larger research effort by researchers at SRTC and Oak Ridge National Laboratory. Information obtained from this effort will help provide information to help mitigate U-rich scale formation during the evaporation and handling of HLW at the SRS. 
WSRC-TR-2002-00510, REVISION 0

\subsection{MATERIALS AND EXPERIMENTAL METHODS}

\subsection{Sample Preparation}

The NAS solids (amorphous zeolite, sodalite and zeolite A) were synthesized according to modified methods supplied by Professor A. Mensah of the University of South Australia. The amorphous zeolite and sodalite were each synthesized in $0.4 \mathrm{M} \mathrm{Al}$ and $0.4 \mathrm{M}$ Si solutions in 4.0 $\mathrm{M} \mathrm{NaOH}$ whereas the zeolite A was synthesized in $0.29 \mathrm{M} \mathrm{Si}$ and $0.29 \mathrm{M}$ Al solutions in $4.7 \mathrm{M}$ $\mathrm{NaOH}$. All NAS solids were synthesized in one-L solutions in the presence of $50 \mathrm{mg}$ U(VI). The difference between the amorphous zeolite and sodalite syntheses was temperature, in that the amorphous zeolite was made at 40 degrees $\mathrm{C}$ and the sodalite was made at 80 degrees $\mathrm{C}$. Zeolite A was made at 90 deg. C. Additional details of the syntheses and the solids characterization are reported by Mensah et al. (2002) and Oji and Williams (2002). ${ }^{29,30}$

After preparation, the solids were washed three times in DI water, filtered with a 0.25 micron nylon filter, and dried in air. The air-dried solids were then washed in three times with $0.4 \mathrm{M}$ sodium carbonate, filtered with a 0.25 micron nylon filter, and then air-dried. The air-dried solids were then provided to us for XAFS analyses. Sub-samples of the solids were digested to determine the $U$ concentrations after synthesis (using inductively-coupled argon plasma mass spectrometry) after each of the two washing steps. The results of the sample digests are shown in Table 3-1. Washing of the U-NAS solids with DI water and carbonate solutions resulted in the removal of salts and NAS material. Hence, in most cases, more NAS was removed by washing (on a percent basis) than the $U$. This preferential removal of $U$ resulted in higher $U$ concentrations ( $U$ enrichment) in the solids after washing - for the amorphous zeolite and the sodalite samples.

The washes of the U-NAS solids were performed to determine the lability of the U that was associated with the NAS solids and to investigate the speciation of $U$ in the resultant washed material. With washing, the more labile (i.e., readily soluble or rapidly dissolving and desorbed $\mathrm{U}$ ) forms of $\mathrm{U}$ are removed, leaving the more stable (less soluble or kinetically slow to dissolve) forms of $U$ in the solid phase. It was anticipated that by selectively washing/leaching various types of $U$ from the solids, a more homogeneous population of $U$ species would be left in the washed solids. Because these U-NAS precipitates did not exhibit any diffraction patterns indicative of known U solids, ${ }^{30}$ XAFS was a suitable technique to use for structural elucidation.

To determine when the $U$ should be added during these NAS syntheses, precipitation timing studies were done with $\mathrm{U}, \mathrm{Al}$ and caustic salt solutions using the same experimental conditions (such as temperature) as those required for the individual NAS syntheses - as discussed in Oji and Williams (2002). ${ }^{30}$ No Si was added to avoid making NAS for each of these reference U materials. The $\mathrm{U}$ added to these solutions underwent precipitation and the unwashed solids were supplied for XAFS analyses. The solids in these "reference" samples may be representative of solids that can form in a heated caustic solutions that are low in Si but contain high Al, such as the uranyl oxyhydroxides. 


\subsection{EXAFS Dat a Collection}

The XAFS data were collected on beamline X23a2 (Figure 3.1) at the National Synchrotron Light Source (NSLS, Brookhaven National Laboratory, Upton, NY). Uranium-XAFS data were collected at the $\mathrm{U} \mathrm{L}_{3}$-edge $(17,166 \mathrm{eV})$ on the air-dried filtered U-containing NAS solids (listed in Table 3-1). The XAFS data were collected in fluorescence mode using an unfocussed X-ray beam and a fixed-exit $\mathrm{Si}(311)$ monochromator (X23a2). Ion chambers were used to collect incident $(I o)$, transmission (It) and reference (Ir) signals. Gas ratios for the data collection in Io were $100 \%$ Ar. A Lytle detector was used to collect fluorescence X-rays (If). The monochromator energy was maximized using a piezo stack feedback energy stabilization system, with a settling time of 0.3 seconds per change in monochromatic energy. An X-ray beam size of 2 by $28 \mathrm{~mm}^{2}$ was used. Energy calibration was done using foils of $\mathrm{Pt}\left(\mathrm{L}_{1}\right.$-edge of 13,880 eV), $\mathrm{Zr}$ (K-edge, 17,998 eV), and Mo (K-edge, 20,000 eV).

Table 3-1 The U-loaded NAS samples that were analyzed with XAFS based on ICP-MS determinations from Oji and Williams (2002).

\begin{tabular}{|c|c|}
\hline Sample Description & $\begin{array}{l}\text { Uranium Concentration } \\
\text { (mg U kg-1 solid after } \\
\text { preparation or washing) }\end{array}$ \\
\hline Unwashed Amorphous Zeolite Material & 280 \\
\hline DI Water Washed Amorphous Zeolite Material & 490 \\
\hline Carbonate Washed Amorphous Zeolite Material & Not determined \\
\hline Unwashed Sodalite & 820 \\
\hline DI Washed Sodalite & 1,500 \\
\hline Carbonate Washed Sodalite & Not determined \\
\hline Unwashed Zeolite A & 180 \\
\hline DI Water Washed Zeolite A & 110 \\
\hline Carbonate Washed Zeolite A & Not determined \\
\hline U Reference Solid for Amorphous Zeolite & Not determined* \\
\hline U Reference Solid for Sodalite & Not determined \\
\hline U Reference Solid for Zeolite A & Not determined \\
\hline
\end{tabular}

* We estimate that these solids contained several percent U based on the high U-XAFS signal that we obtained for them. 


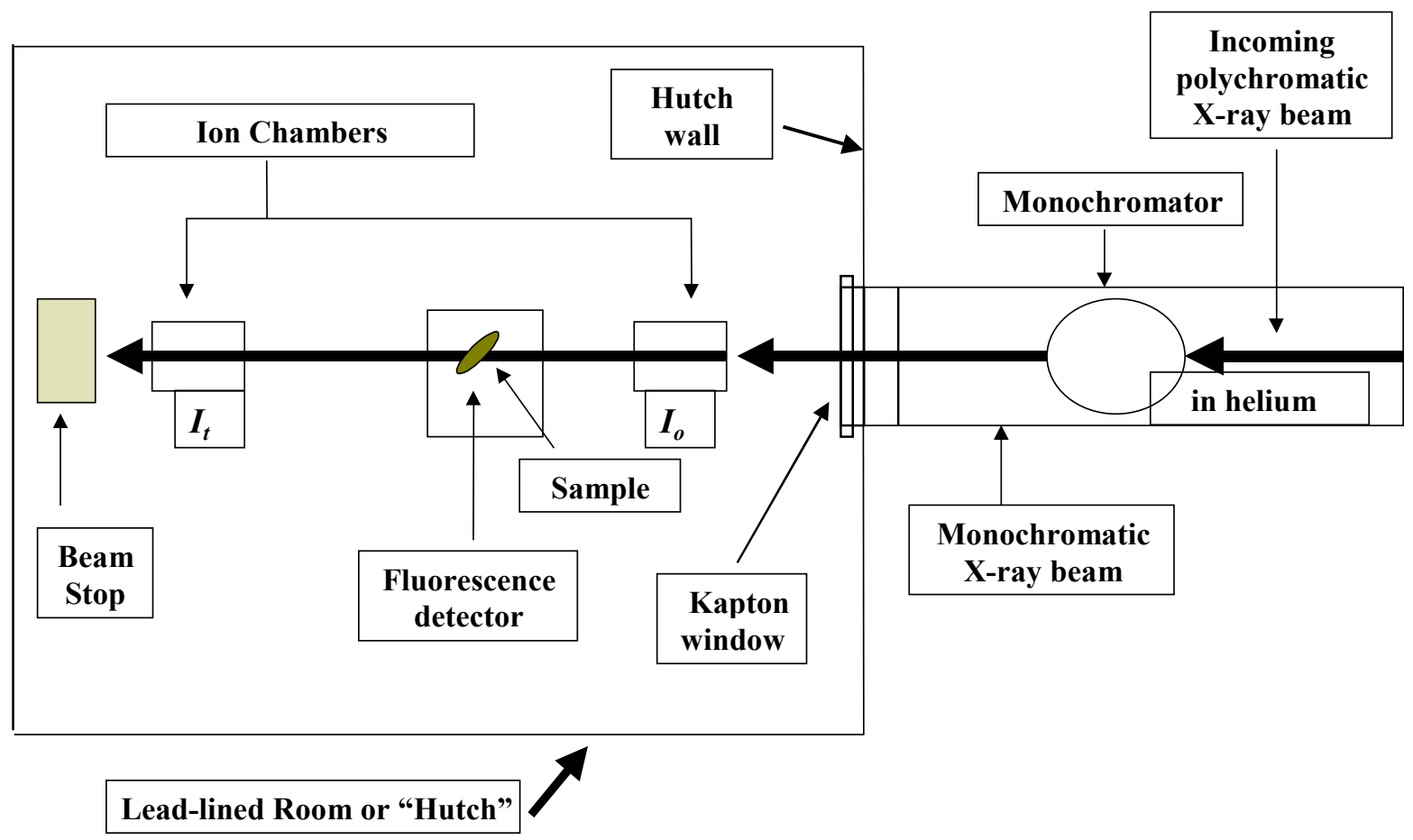

Figure 3.1 Diagram of the downstream portion of the hutch table at NIST beamline X23a2 (not drawn to scale).

\subsection{XANES and EXAFS Data Analyses}

The background contribution to the EXAFS spectra was removed using an algorithm (AUTOBK) developed by Newville et al. (1993), which minimizes R-space values in low $k$ space. Each chi data set was read into the WINXAS analysis package. ${ }^{31,32}$ Replicate scans were co-added to improve $\mathrm{S} / \mathrm{N}$. After background subtraction and normalization, the U XANES spectra were compared with spectra from the U standard. The U-XAFS spectra were analyzed from 2 to $12 \AA^{-1}$. The chi data were $k^{3}$-weighted and Fourier-transformed (FT) to yield R-space or Radial Distribution Function (RDF) plots. ${ }^{33}$ Simulated EXAFS spectra were also generated based on the documented crystallographic properties for uranyl silicate and oxyhydroxide solids using ab initio based theory, which involved FEFF 7.2 a program created by researchers at the Univ. of Washington. . $^{\text {35, 36, 37, 38, } 39}$ Model fits for U were performed in R-space. 
WSRC-TR-2002-00510, REVISION 0

\subsection{RESULTS}

\subsection{Background on the Characterization of U Behavior on Surfaces with XAFS}

The XAFS techniques have been applied to the study of U(VI) on a variety of solids, such as Fe oxides, carbonates, silicates and sulfides $40,41,42,43,44,45,46,47,48$ and within mineral oxides, calcite, oxyhydroxides and gels. ${ }^{49,50,51,52,53}$ Most of these studies have an environmental focus because they were performed with naturally-occurring minerals and U-containing minerals under conditions relevant to the geologic surface and subsurface environments. The findings of the sorption studies vary greatly but they typically indicate that sorbed $\mathrm{UO}_{2}{ }^{2+}$ species (in the absence of a redox-active mineral surface) form inner sphere bonds with the sorbent minerals over a solution $\mathrm{pH}$ range between 5 to 12 via primarily edge linking with octahedral or tetrahedral structural components. A list of the results for many the XAFS studies on sorbed U species is shown in Table 4.1. Corner linking in addition to edge linking of sorbed $U$ is observed at the muscovite surface, however, this aluminosilicate has a difference structure than that of zeolites. The surface sites on zeolites offer corner linking sites through single terminal $\mathrm{O}$ atoms, as opposed to edge linking sites, which offer edge linking via two $\mathrm{O}$ atoms.

The U(VI)-complexes that have been observed on surfaces using XAFS techniques are typically monomeric $\mathrm{U}(\mathrm{VI})$-hydroxo and $\mathrm{U}(\mathrm{VI})$-carbonato species that form mono- and bidentate linkages with the participating surfaces (as noted in Fig. 4.1 and Table 4.1). In acidic solutions, some U(VI) species sorb mainly via an outer sphere mechanism on some silicates. Dimeric U(VI) species are also observed. XAFS and wet chemical studies have elucidated that U(VI) sorbs on broken edge sites of layered smectitic (aluminosilicate clay) minerals. These aluminosilicate minerals typically sorb cations such as $\mathrm{Na}^{+}$and $\mathrm{Ca}^{2+}$, which form outer sphere (electrostatic) surface complexes on the fixed charge sites of basal planes of edge-sharing Al octahedra and edge-sharing Si tetrahedra as shown in Figure 4.1. ${ }^{54}$ The broken edge sites of these minerals, which possess $\mathrm{pH}$-dependent charge are thought to host the sorption of U(VI) species. ${ }^{55,56} \mathrm{In}$ contrast, XAFS and X-ray diffraction crystallographic studies have shown that uranyl silicate minerals such as soddyite $\left[\left(\mathrm{UO}_{2}\right)_{2} \mathrm{SiO}_{4} 2 \mathrm{H}_{2} \mathrm{O}\right], \mathrm{Na}_{4}\left(\mathrm{UO}_{2}\right)_{2} \mathrm{Si}_{4} \mathrm{O}_{10} \cdot 4 \mathrm{H}_{2} \mathrm{O}_{(\mathrm{s})}$, Na-boltwoodite $\left[\mathrm{Na}_{2}\left(\mathrm{UO}_{2}\right)_{2}\left(\mathrm{SiO}_{3} \mathrm{OH}\right)_{2} \cdot 1.5 \mathrm{H}_{2} \mathrm{O}\right]$ and Na-weeksite $\left[\mathrm{Na}_{2}\left(\mathrm{UO}_{2}\right)_{2}\left(\mathrm{Si}_{5} \mathrm{O}_{13}\right)_{6} \cdot 3 \mathrm{H}_{2} \mathrm{O}\right]$ possess predominantly corner-sharing behavior as opposed to edge sharing behavior between $\mathrm{U}$ polyhedra and silica tetrahedra as shown in Figure 4.2.

Little if any experimental XAFS work has been done with dissolved, sorbed $\mathrm{U}$ or precipitated species under conditions relevant to that of HLW solutions. However, the hexavalent U(VI) is probably the predominant oxidation state of U in HLW salt solutions. Uranium-XAFS studies have been performed to study the structure of high levels of dissolved U(VI) species under simulated caustic solutions [in $3.5 \mathrm{M}$ tetramethyl-ammonium, which was used as a counterion to suppress precipitation of $\mathrm{U}(\mathrm{VI})]^{60}$ and of solid phase $\mathrm{U}$ in real HLW samples. ${ }^{61}$ These XAFS studies identified that the most common $\mathrm{U}(\mathrm{VI})$ complexes in caustic solutions are likely to be the monomeric $\mathrm{UO}_{2}(\mathrm{OH})_{4}{ }^{2-}$ and $\mathrm{UO}_{2}(\mathrm{OH})_{5}{ }^{-}$species. Our recent studies with $\mathrm{U}$ in real $\mathrm{HLW}$ sludge indicate that about $95 \%$ of the sludge $\mathrm{U}$ exists in the hexavalent $\mathrm{U}(\mathrm{VI})$ form whereas about $5 \%$ of the $\mathrm{U}$ is metallic. ${ }^{61}$ 


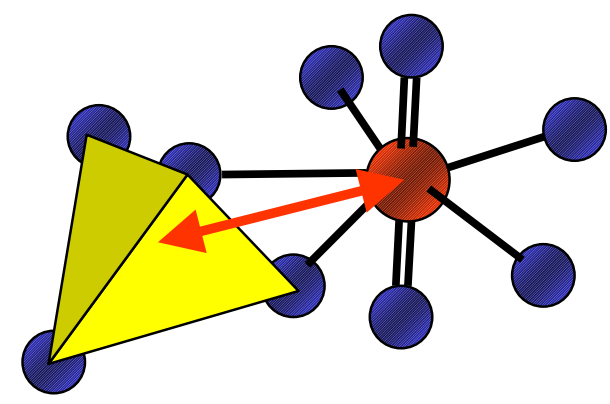

In all published U-EXAFS literature on uranyl sorption to silica gels, the U-Si distance is $\sim 2.71 \AA$ (red arrow), and has been interpreted as "edge sharing".

Hence, short U-Si distances WITHOUT the presence of long radial distances is a signature for sorption

Legend:

uranium
oxygen
silicon

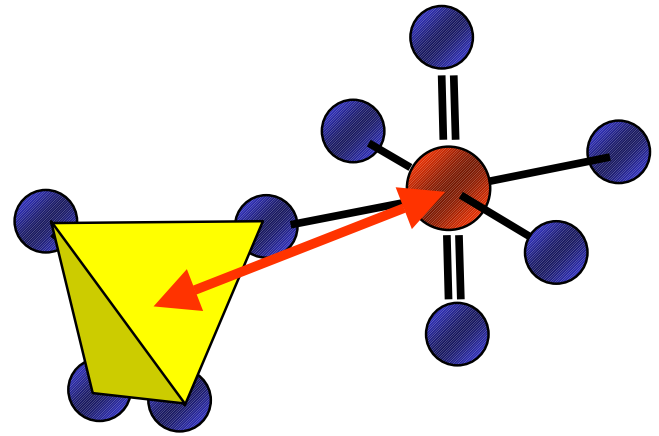

In published U-EXAFS literature on uranyl silicates, the U-Si distance is $\sim 3.7 \AA$ (red arrow) indicative of "corner sharing" or near $\sim 3.2 \AA$, which is indicative of edge sharing (not shown).

Long U-Si distances is a signature for structural incorporation of $U$

Figure 4.1 Pictorial representation of U(VI) edge and corner sharing with silica tetrahedra. 
Table 4-1 Literature XAFS fit data for sorbed U(VI) on characterized Al and Si minerals. The magnitude of the Debye-Waller Factor $\left[\sigma^{2}[\AA]^{2}\right]$ indicates the variation in the bond length determination.

\begin{tabular}{|c|c|c|c|c|}
\hline $\begin{array}{c}\text { Sample (with Mechanism } \\
\text { of Sorption) }\end{array}$ & Interaction & $\mathbf{C N}$ & $\begin{array}{c}\text { Distance } \\
\mathbf{R}[\AA]]\end{array}$ & $\sigma^{2}[\AA]^{2}$ \\
\hline \multirow{5}{*}{$\begin{array}{l}0.4 \mathrm{mM} \mathrm{U}(\mathrm{VI}) \text { sorbed to muscovite (an } \\
\text { layered aluminosilicate, } \mathrm{pH} 4.6)^{40,} * \\
\text { (Inner sphere) }\end{array}$} & $\mathrm{U}-\mathrm{O}_{\mathrm{ax}}$ & 2 & 1.80 & 0.003 \\
\hline & $\mathrm{U}-\mathrm{O}_{\mathrm{eq}}$ & 4 & 2.35 & 0.028 \\
\hline & $\mathrm{U}-\mathrm{Si}_{1}$ & 1 & 2.75 & 0.011 \\
\hline & $\mathrm{U}-\mathrm{Si}_{2}$ & 2 & 3.66 & 0.010 \\
\hline & U-U & 1 & 3.86 & 0.010 \\
\hline \multirow{6}{*}{$\begin{array}{l}\text { Natural U-rich FeSi oxide material } \\
\text { (Inner sphere) }\end{array}$} & $\mathrm{U}-\mathrm{O}_{\mathrm{ax}}$ & 2.1 & 1.80 & 0.005 \\
\hline & $\mathrm{U}-\mathrm{O}_{\mathrm{eq} 1}$ & 1.8 & 2.32 & 0.005 \\
\hline & $\mathrm{U}-\mathrm{O}_{\mathrm{eq} 2}$ & 1.8 & 2.48 & 0.004 \\
\hline & $\mathrm{U}-\mathrm{Si}_{1}$ & 0.1 & 3.29 & 0.001 \\
\hline & $\mathrm{U}-\mathrm{Si}_{2}$ & 4.0 & 3.67 & 0.020 \\
\hline & U-U & 1.6 & 3.81 & 0.009 \\
\hline \multirow{3}{*}{$\begin{array}{l}\text { Silica gel, } 28 \mathrm{mg} \mathrm{U} \mathrm{kg}^{-1} \text { solid, } \mathrm{pH} 4^{46} \\
\text { (Outer sphere) }\end{array}$} & $\mathrm{U}-\mathrm{O}_{\mathrm{ax}}$ & 2.0 & 1.80 & 0.015 \\
\hline & $\mathrm{U}-\mathrm{O}_{\mathrm{eq} 1}$ & 2.2 & 2.29 & 0.0057 \\
\hline & $\mathrm{U}-\mathrm{O}_{\mathrm{eq} 2}$ & 3.0 & 2.51 & 0.0070 \\
\hline \multirow{3}{*}{$\begin{array}{l}\text { Silica gel, } 59 \mathrm{mg} \mathrm{U}(\mathrm{VI}) \mathrm{kg}^{-1} \text { solid, } \mathrm{pH} \sim 4 \\
\text { (Outer sphere) }\end{array}$} & $\mathrm{U}^{-\mathrm{O}_{\mathrm{ax}}}$ & 2.00 & 1.80 & 0.0150 \\
\hline & $\mathrm{U}-\mathrm{O}_{\mathrm{eq} 1}$ & 2.29 & 2.29 & 0.0057 \\
\hline & $\mathrm{U}-\mathrm{O}_{\mathrm{eq} 2}$ & 2.51 & 2.51 & 0.0070 \\
\hline \multirow{3}{*}{$\begin{array}{l}\text { Silica gel, } 0.5 \mathrm{mg} \mathrm{U}(\mathrm{VI}) \mathrm{kg}^{-1} \text { solid, } \mathrm{pH} \\
4.5^{62} \\
\text { (Outer sphere) }\end{array}$} & $\mathrm{U}-\mathrm{O}_{\mathrm{ax}}$ & 2.0 & 1.80 & 0.0013 \\
\hline & $\mathrm{U}-\mathrm{O}_{\mathrm{eq}}$ & 2.26 & 2.29 & 0.0035 \\
\hline & U-Si & 0.50 & 2.72 & 0.0034 \\
\hline \multirow{3}{*}{$\begin{array}{l}\text { Hydrobiotite, } 1.1 \mathrm{mM} \mathrm{U}(\mathrm{VI}), \mathrm{pH} \sim 2.5^{4 I} \\
\text { (Outer sphere) }\end{array}$} & $\mathrm{U}-\mathrm{O}_{\mathrm{ax}}$ & 2.03 & 1.78 & 0.0012 \\
\hline & $\mathrm{U}-\mathrm{O}_{\mathrm{eq} 1}$ & 3.76 & 2.39 & 0.0087 \\
\hline & $\mathrm{U}-\mathrm{O}_{\mathrm{eq} 2}$ & 0.09 & 2.51 & 0.0002 \\
\hline \multirow{3}{*}{$\begin{array}{l}\text { Hydrobiotite, } 3.3 \mu \mathrm{M} \mathrm{U}(\mathrm{VI}), \mathrm{pH} \sim 6-7^{41} \\
\text { (Outer sphere) }\end{array}$} & $\mathrm{U}-\mathrm{O}_{\mathrm{ax}}$ & 2.10 & 1.79 & 0.0012 \\
\hline & $\mathrm{U}-\mathrm{O}_{\mathrm{eq} 1}$ & 1.27 & 2.24 & 0.0076 \\
\hline & $\mathrm{U}-\mathrm{O}_{\mathrm{eq} 2}$ & 3.09 & 2.42 & 0.0060 \\
\hline \multirow{2}{*}{$\begin{array}{l}\text { Vermiculite, } 1.1 \mathrm{mM} \mathrm{U}(\mathrm{VI}), \mathrm{pH} \sim 2.5^{41} \\
\text { (Outer sphere) }\end{array}$} & $\mathrm{U}-\mathrm{O}_{\mathrm{ax}}$ & 2.69 & 1.77 & 0.0034 \\
\hline & $\mathrm{U}-\mathrm{O}_{\mathrm{eq}}$ & 3.52 & 2.43 & 0.0090 \\
\hline \multirow{4}{*}{$\begin{array}{l}\text { Vermiculite, } 3.3 \mu \mathrm{M} \mathrm{U}(\mathrm{VI}), \mathrm{pH} \sim 6-7^{41} \\
\text { (Outer sphere) }\end{array}$} & $\mathrm{U}-\mathrm{O}_{\mathrm{ax}}$ & 2.23 & 1.80 & 0.0032 \\
\hline & $\mathrm{U}-\mathrm{O}_{\mathrm{eq} 1}$ & 3.09 & 2.28 & 0.0115 \\
\hline & $\mathrm{U}-\mathrm{O}_{\mathrm{eq} 2}$ & 1.02 & 2.48 & 0.0023 \\
\hline & U-U & 0.63 & 3.88 & 0.0023 \\
\hline
\end{tabular}

* The local environment of sorbed U on muscovite was described using U-XAFS data and modeled by Moyes et al. (2000). They observed edge sharing between the uranyl and a Si tetrahedron (i.e., the shortest U-Si radial distance). The longer U-Si distance was obtained by positioning a second neighboring corner sharing Si tetrahedron next to the first edge sharing $\mathrm{Si}$ tetrahedron. Hence, bonding was primarily through the edge sharing Si tetrahedron that was also bonded to another Si tetrahedron. 
Uranium bearing silicate minerals are characterized by $\mathrm{U}$ cross-linked by corner sharing $\mathrm{SiO}_{4}$ tetrahedra as well as some edge sharing $\mathrm{SiO}_{4}$ tetrahedra. The local environment of the $\mathrm{U}$ in the structures below has 5 equatorial $\mathrm{O}$ atoms.

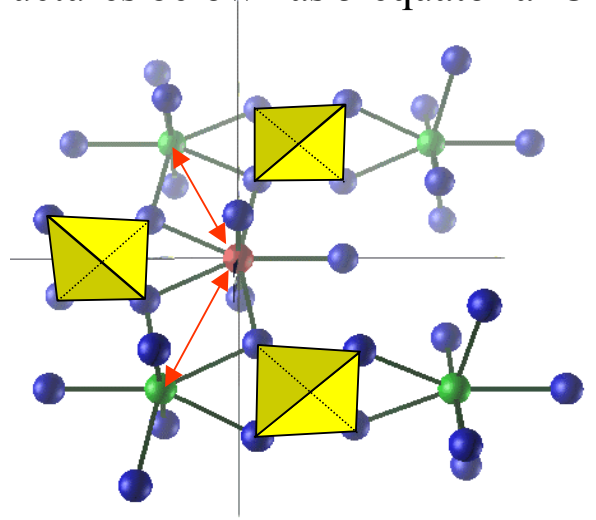

Soddyite: one edge sharing, two corner sharing $\mathrm{SiO}_{4}$ tetrahedra

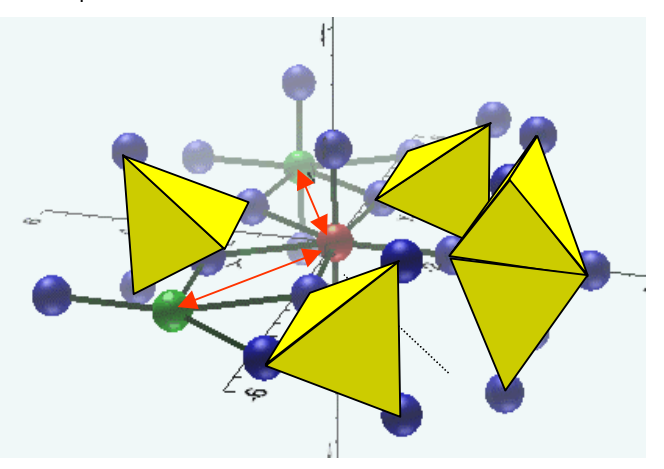

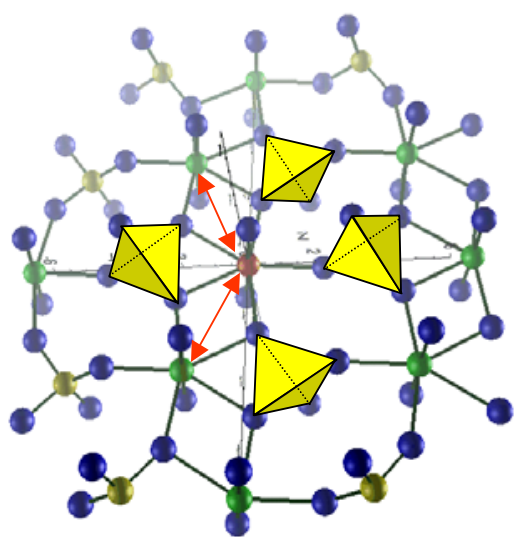

Boltwoodite: one edge sharing, three corner sharing $\mathrm{SiO}_{4}$ tetrahedra

Legend:

central uranium

oxygen

silicon

neighboring uranium

Weeksite: one edge sharing, four corner sharing $\mathrm{SiO}_{4}$ tetrahedra

Figure 4.2 Examples of uranyl silicate structures. Red arrows denote radial U-U distances that can be obtained using crystallographic and XAFS analyses. 


\subsection{EXAFS Analyses}

In simple terms, chi data (the plot of the wavevector in reciprocal space) show the oscillatory component (with both constructive and destructive interferences) of the atoms in the neighbor environment of the element of interest. The chi data represent part of the photoelectron wave that can be defined by the EXAFS equation. ${ }^{26,27,63}$ The EXAFS equation is shown in a highly simplified form below (see list of definitions for explanation of equation terms):

$$
\operatorname{Chi}(\mathrm{k})=\frac{\mathrm{F}(\mathrm{k}) * \mathrm{~N} * \mathrm{~S}}{\mathrm{k}^{*} \mathrm{R}^{2}} S_{0}^{2} e^{\left(-2 * \mathrm{k}^{3} \sigma^{2}\right)} \sin [2 * \mathrm{k} * \mathrm{R}+\delta(\mathrm{K})] .
$$

\subsubsection{Uranium Ch i Data for U(VI)-Loaded NAS U Reference Samples}

\subsubsection{Amorphous Zeolite Precursor Samples and the Si-free Reference Sample for the Amorphous Zeolite Syntheses}

The $k^{3}$-weighted chi spectra for the $\mathrm{U}$ in the $\mathrm{U}$ reference and the various washed and unwashed U-containing amorphous zeolite precursor materials are shown in Figure 4.3. The chi data for $U$ in these samples indicate there are light and heavy atom backscatterers as evidenced by the presence of a large amplitude signals at low and high $\mathrm{k}$. In general, the spectra show many similar features - particularly at low k.

\subsubsection{Sodalite Samples and the Si-free Reference Sample for the Sodalite Syntheses}

The $k^{3}$-weighted chi spectra for the $\mathrm{U}$ in the $\mathrm{U}$ reference and $\mathrm{U}$-containing amorphous sodalite precursor material are shown in Figure 4.4. The chi data for $U$ in these samples indicate there are light and heavy atom backscatterers as evidenced by the presence of a large amplitude signals at low and high $\mathrm{k}$. With the exception of the unwashed zeolite, the spectra are quite similar.

\subsubsection{Si-free Refer ence Sample for the Zeolite A Syntheses}

The zeolite samples did not contain sufficient U concentrations for XAFS data collection so no XAFS analyses with these samples are reported. The $k^{3}$-weighted chi spectra for the $U$ in the $U$ reference and U-containing amorphous zeolite precursor material are shown in Figure 4.5. The chi data for $U$ in the $U$ reference material has one or more heavy atom backscatterers as evidenced by the presence of a large amplitude signal at high $\mathrm{k}$. The $\mathrm{U}$ concentrations in the unwashed and washed U-loaded zeolite A samples were not high enough for suitable XAFS data acquisition. 


\section{U Reference and Amorphous Zeolite Precursor Material}

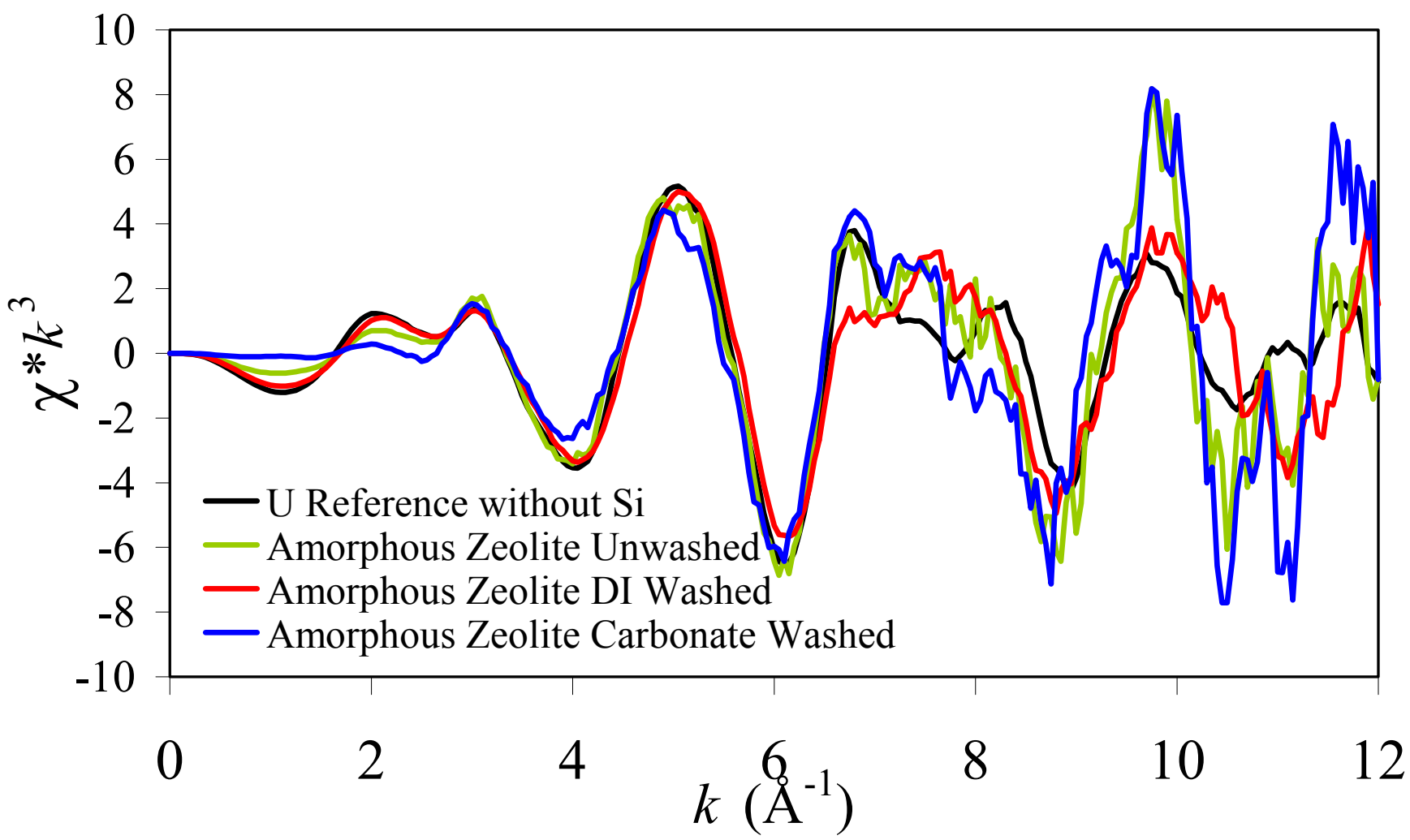

Figure 4.3 The $k^{3}$-weighted chi data (the plot of the wavevector in reciprocal space) for the amorphous zeolite samples and $\mathrm{Si}$-free $\mathrm{U}$ reference samples. 
U Reference and Sodalite Series

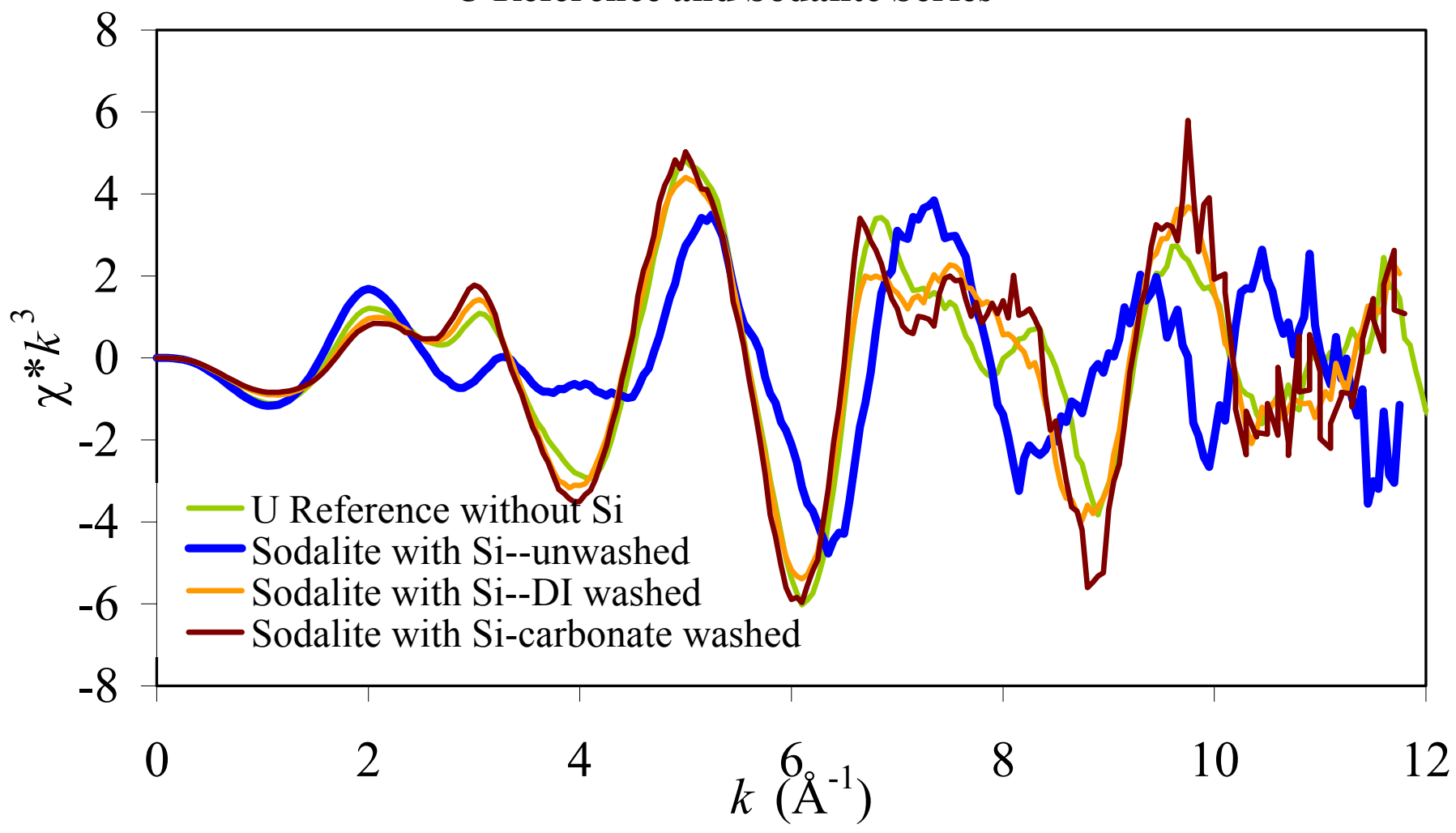

Figure 4.4 The $k^{3}$-weighted chi data (the plot of the wavevector in reciprocal space) for the sodalite samples and the $\mathrm{Si}$-free $\mathrm{U}$ reference sample. 


\section{U Reference for Zeolite A}

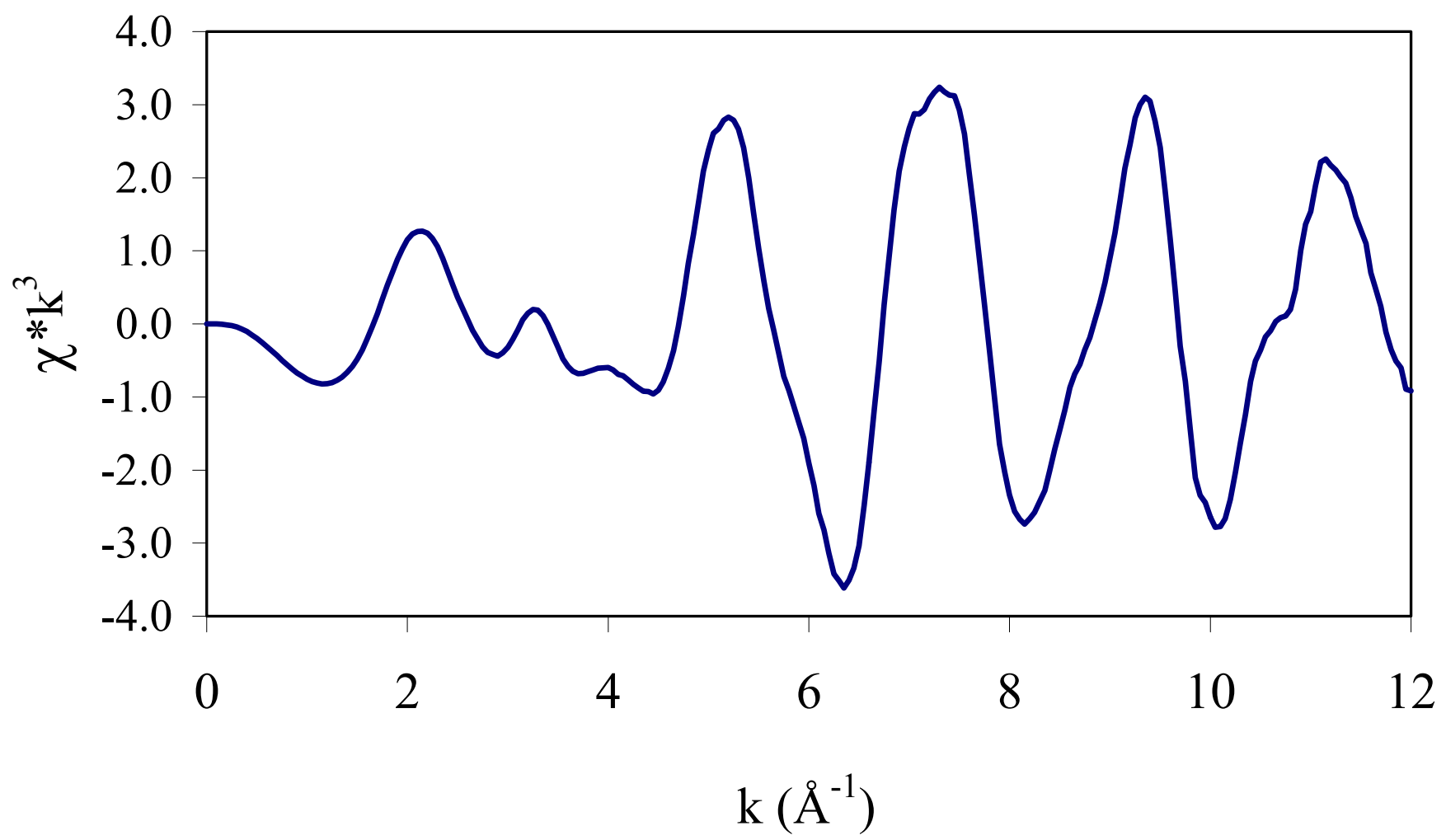

Figure 4.5 The $k^{3}$-weighted chi data (the plot of the wavevector in reciprocal space) for the Sifree zeolite A U reference sample. 
WSRC-TR-2002-00510, REVISION 0

\subsubsection{Uranium Fo urier-Transformed Data}

\subsubsection{Fourier-Transformed Data for $U$ in the U(VI)-containing Amorphous Zeolite and Corresponding Si-free U Reference Samples}

The Fourier-Transformed (FT) data for the amorphous zeolite series samples are shown in Figure 4.6. For all of the samples, the first coordination shell consists of a split uranyl-type shell that contains $\mathrm{O}$ atoms at two different distances. These two first shell peaks represent axial and equatorial U-O bonding behavior as shown pictorially in Fig. 4.7. There are significant peaks in the transforms at greater radial distances (i.e., outer shells) for the unwashed and carbonatewashed amorphous zeolite solids. The FT data for the DI-washed amorphous zeolite sample and the $U$ reference for the amorphous zeolite synthesis do not possess significant higher shell peaks. The higher coordination shell peaks in the FT data for the carbonate-washed sample are more pronounced than that for the DI washed and unwashed samples - suggesting that washing removes one (or more) $U$ phases. Hence, washing may result in a $U$ solid phase that is more characteristic of a single $U$ phase because the magnitude of FT peaks increases with washing. The lack of strongly defined peaks in the 3 to $4 \AA$ region of the unwashed and DI-washed amorphous zeolite sample spectra is characteristic of the uranyl oxide hydrate called schoepite $\left[\mathrm{U}(\mathrm{VI}) \mathrm{O}_{3} \cdot 2 \mathrm{H}_{2} \mathrm{O}_{(\mathrm{s})}\right] .{ }^{49}$ The XAFS spectra for U-U interactions in schoepite are representative of multiple environments, which results in decreased peak heights in the FT data for the outer shell U-U interactions. Nevertheless, it is difficult to interpret XAFS spectra that are representative of multiple species.

\subsubsection{Fourier-Transformed Data for $U$ in the $U(V I)$-containing Sodalite and Corresponding Si-free U Reference Samples}

The FT data for the sodalite series samples is shown in Figure 4.8. Three of the samples contain a typical split first shell environment for hexavalent $U$ (much like that described for the samples in section 4.2.2.1). These two first shell peaks are indicative of uranyl behavior. The FT data for the fourth sample (the unwashed sodalite sample) contains three peaks in the first coordination shell. The additional (middle distance) peak is indicative of a uranate-type behavior as opposed to uranyl-type behavior as illustrated in Fig. 4.7. Uranate-type bonding behavior is less common than that of uranyl bonding behavior. ${ }^{64,65,66,67}$ Additionally, uranate type bonding is not common to all uranate solids. Some uranate solids possess uranyl behavior and some do not. $^{68}$ It is thought that sodium diuranate has a uranyl-type environment. ${ }^{64}$

As was observed with the FT data from the amorphous zeolite U reference spectra, the outer shell peaks in the FT data for the carbonate-washed sodalite sample are more pronounced than that for the DI washed and unwashed sodalite samples - suggesting that washing removes one (or more) $U$ phases. Hence, washing may result in a $U$ solid that is more characteristic of a single $U$ phase because the magnitude of FT peaks increases with washing. 


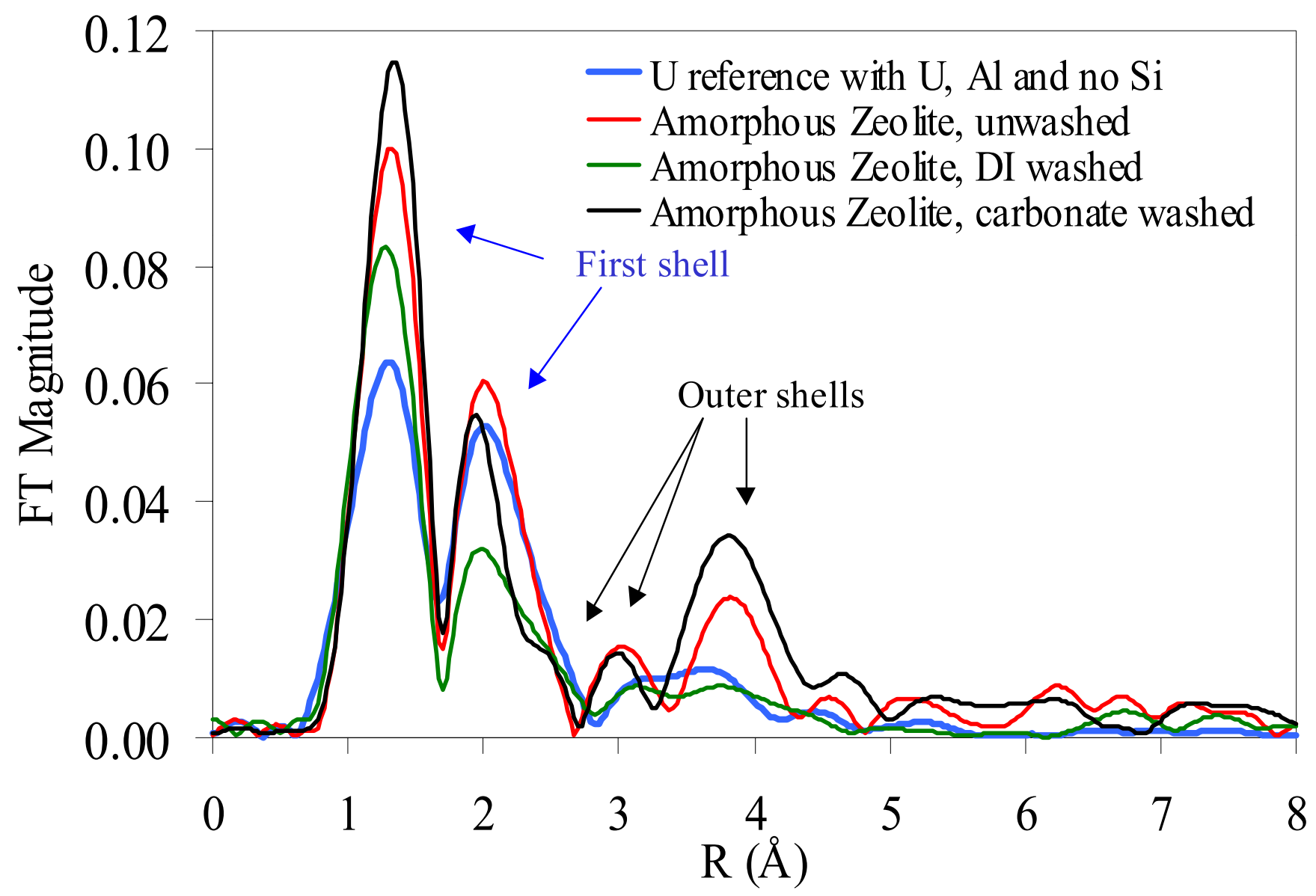

Figure 4.6 FT data for the amorphous zeolite series samples—uncorrected for phase shift. 


\section{Uranyl First Shell Structure}

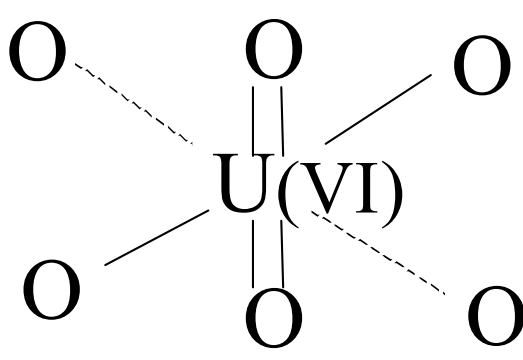

- 2 axial oxygen $(\mathrm{O})$ bonds at $\sim 1.75-1.8 \AA$.

- 4 (or more) equatorial $\mathrm{O}$ bonds at $\sim 2.25-2.48 \AA$.

- Common form of $U$.

- Example: $\mathrm{Na}_{4} \mathrm{UO}_{2}\left(\mathrm{CO}_{3}\right)_{3(\mathrm{~s})}$.
Uranate First Shell Structure

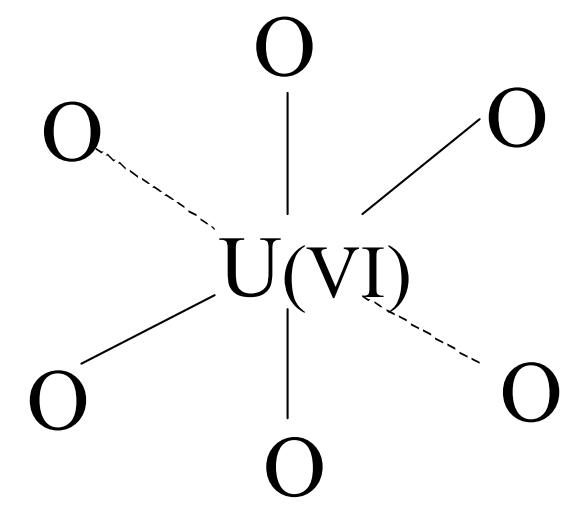

- 3 or more O bonds at $\sim 2.1-2.3 \AA$.

- Forms at temperature, structures poorly known.

- Aqueous solubility: < 100 ppm U but soluble in carbonate/acidic solutions. - $U$ present as $\mathrm{U}^{6+}$ ion [0.52-0.86 $\mathrm{A}$. - Examples: $\mathrm{Ba}_{3} \mathrm{PuO}_{6(\mathrm{~s})}, \mathrm{CoUO}_{4(\mathrm{~s})}$, glasses. Farges et al. 1992; Shannon 1976; Griffiths \& Volkovich, 1999.

Figure 4.7 The two types of U(VI) first shell bonding environments. ${ }^{64,65,66}$ The solids with uranate in their names such as sodium diuranate consist of uranyl-type bonding. Hence, the name of the solid does not always describe the nature of the bonding. 


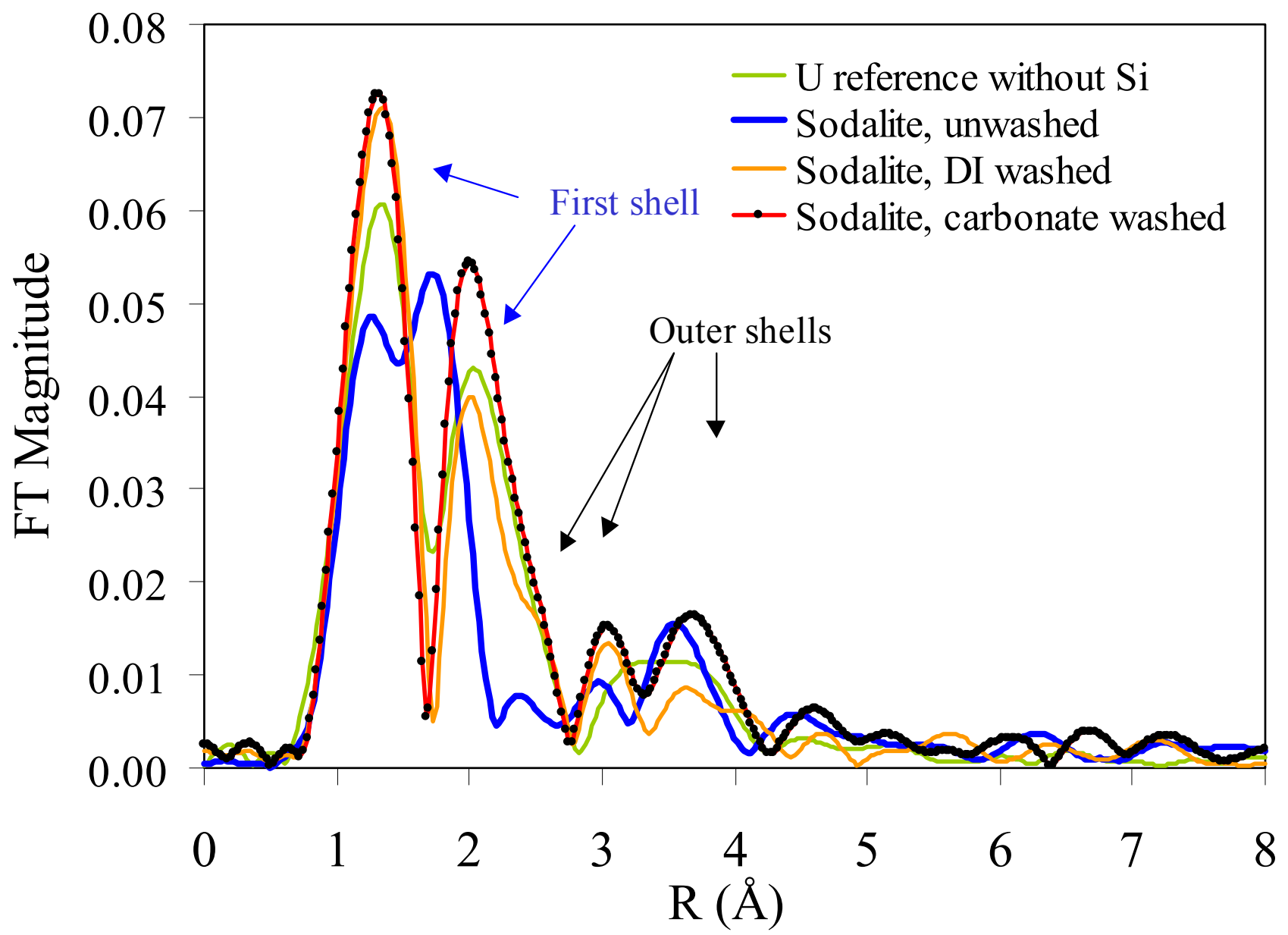

Figure 4.8 FT data for the sodalite series samples - uncorrected for phase shift. 


\subsubsection{Uranium FT and Model Fit Data}

Due to the presence of multiple local environments in most of the XAFS spectra for the U samples, fits were only performed with the spectra for the three $U$ reference samples and the two carbonate-washed amorphous zeolite and sodalite samples. The complexities associated with XAFS analyses for samples with multiple environments will be discussed in the following sections. As discussed previously, XAFS analyses provide average coordination and bonding environment information and they typically cannot provide information on population distributions of species.

\subsubsection{Fourier-Transformed Data for $U$ in the Si-free $U$ Reference Sample for the Amorphous Zeolite Synthesis}

The FT data and corresponding first shell fit data for the Si-free U reference solid (for the amorphous zeolite synthesis) are shown in Fig. 4.9. This $U$ in this sample has two sets of uranyltype $\mathrm{O}$ atoms at $1.76 \AA$ and $1.88 \AA$ and five equatorial $\mathrm{O}$ atoms at $2.42 \AA$. Fits performed with a single set of axial type U-O interactions were not successful. We recognize that having four axial $\mathrm{O}$ atoms at two different distances is uncommon to most $\mathrm{U}$ solids. The complexities associated with these fits suggest that more than three environments exist for the local coordination of $U$ in this sample. Hence, fits for interactions for higher shells were not performed due to the multiple environments.

\subsubsection{Fourier-Transformed Data for $U$ in the Si-free $U$ Reference Sample for the Sodalite Synthesis}

The FT data and corresponding first shell fit data for the Si-free U reference solid (for the sodalite synthesis) are shown in Fig. 4.10. This solid has two sets of uranyl-type axial $\mathrm{O}$ atoms at $1.76 \AA$ and $1.87 \AA$ and four equatorial $\mathrm{O}$ atoms at $2.40 \AA$. These first coordination shell data are similar to that obtained for the $U$ reference solid as discussed in the previous section. Fits that were performed using a single set of axial type U-O interactions were unsuccessful. Again, we recognize that having four axial $\mathrm{O}$ atoms at two different distances is uncommon to most $\mathrm{U}$ solids. The complexities associated with these fits suggest that multiple environments (at least three) exist for the local coordination environment of $U$ in this sample.

Fits were successfully performed for the second shell interactions (Fig. 4.11). Fits for these outer shell interactions indicate the presence of one Al atom in the outer shell at $3.60 \AA$ (based on using $\mathrm{Si}$, which is a good analog for $\mathrm{Al}$ because it has a similar mass to that of $\mathrm{Al}$ ) in addition to three $\mathrm{U}$ atoms at $3.83 \AA$. These data suggest that a U(VI)-aluminate solid phase may be present in this sample. Aging studies conducted by SRTC researchers have been conducted to examine U(VI) solubility in HLW simulant solutions over a large range of hydroxide, nitrate, nitrite, and aluminate concentrations - in addition to two temperatures (room temperature and 85

$\left.{ }^{\circ} \mathrm{C}\right) .{ }^{69,70}$ These studies did not attribute $U$ solubility to be strongly influenced by aluminate concentration. Decreases in aluminate levels were attributed to the precipitation of Alcontaining solids (such as gibbsite). Some of the decreases in U solubility that were observed were thought to be due to multiple component effects, which could not be statistically isolated. However, aluminate concentration was used in the predictive $U$ solubility equations that were 
generated from this work. The solids from these studies were not isolated and characterized. Additional work is recommended to investigate the influence of $\mathrm{Al}$ on $\mathrm{U}$ solubility and the local coordination environments of $\mathrm{U}$ because $\mathrm{U}(\mathrm{VI})$-aluminate solids have not been documented in the literature. These solids may not possess long range order and may have historically (if ever studied) been amorphous to XRD analysis. Our U-XAFS study pertains to non-replicated syntheses and the low levels of $U$ in this sample make conclusions about higher shell behavior less straightforward than that which would be anticipated for studies with pure crystalline $\mathrm{U}$ solids such as that for uraninite $\left[\mathrm{U}(\mathrm{IV}) \mathrm{O}_{2(\mathrm{~s})}\right]$. Such studies with a more structurally pure $\mathrm{U}$ phase would be complex but better fits of the XAFS data would be expected. 


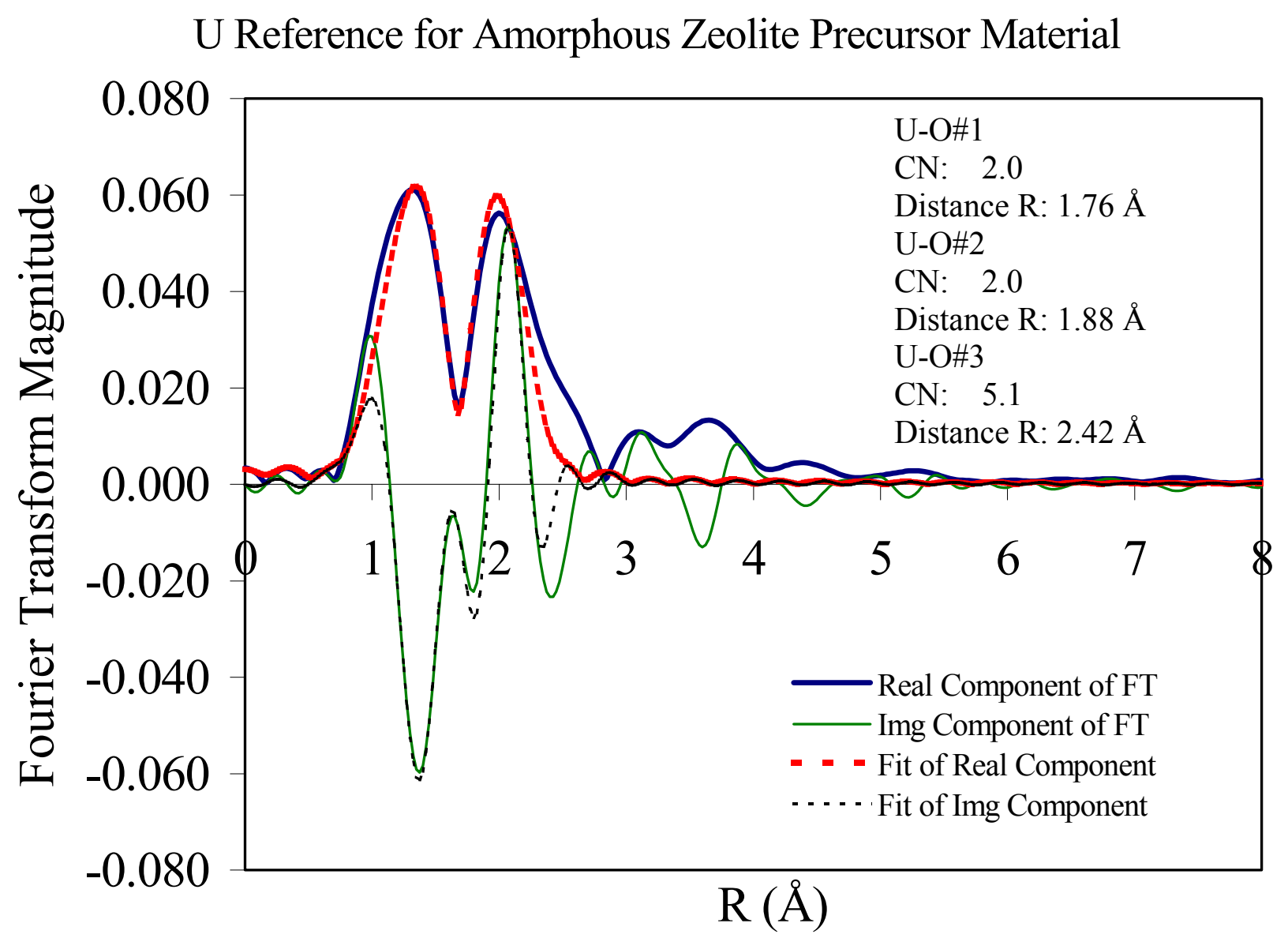

Figure 4.9 FT and first coordination shell fit data for the Si-free U reference (for the amorphous zeolite synthesis) — uncorrected for phase shift. 


\section{U Reference for Sodalite}

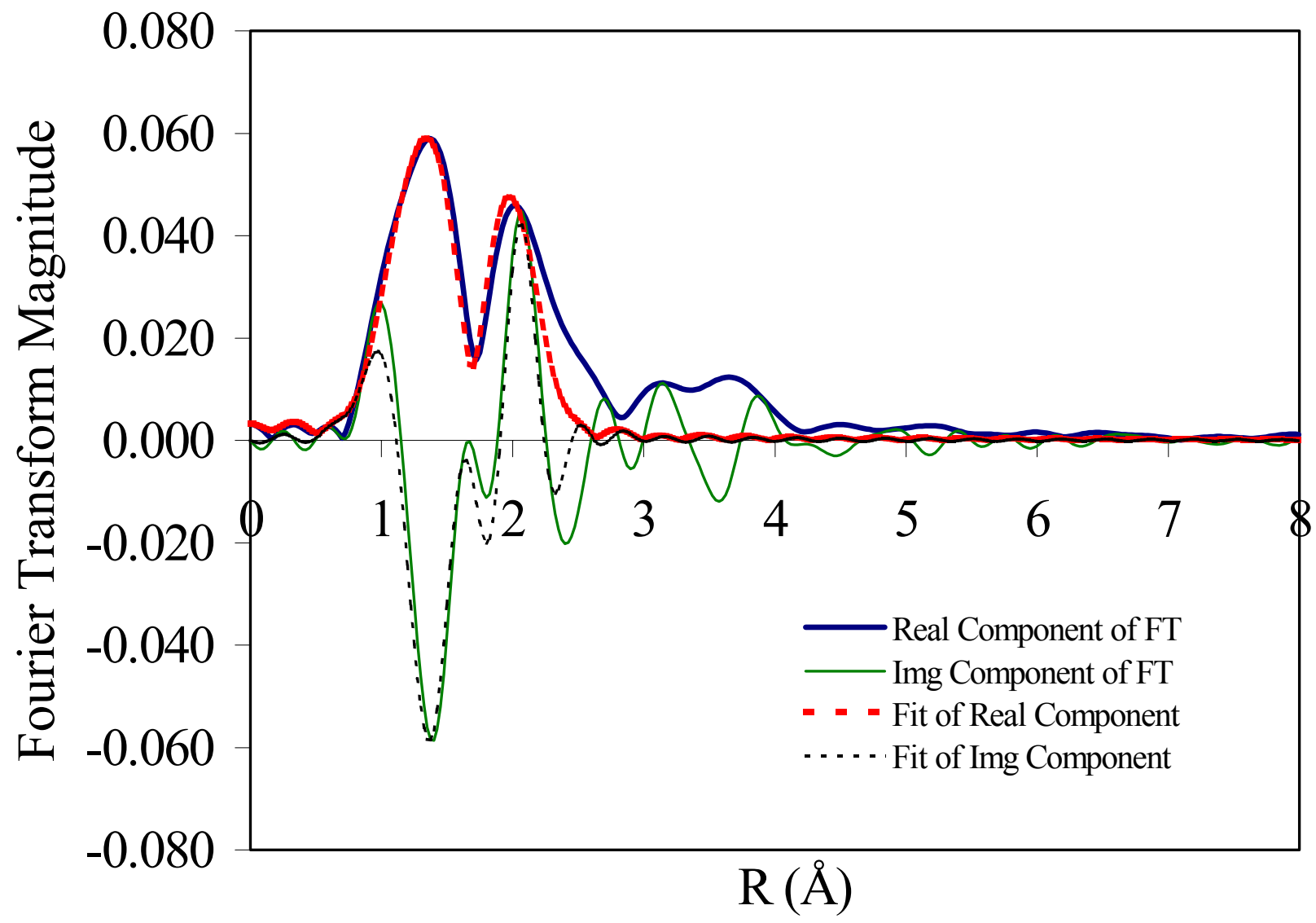

Figure 4.10 FT and first coordination shell fit data for the Si-free U reference sample (for the sodalite synthesis) - uncorrected for phase shift. 


\section{U Reference for Sodalite}

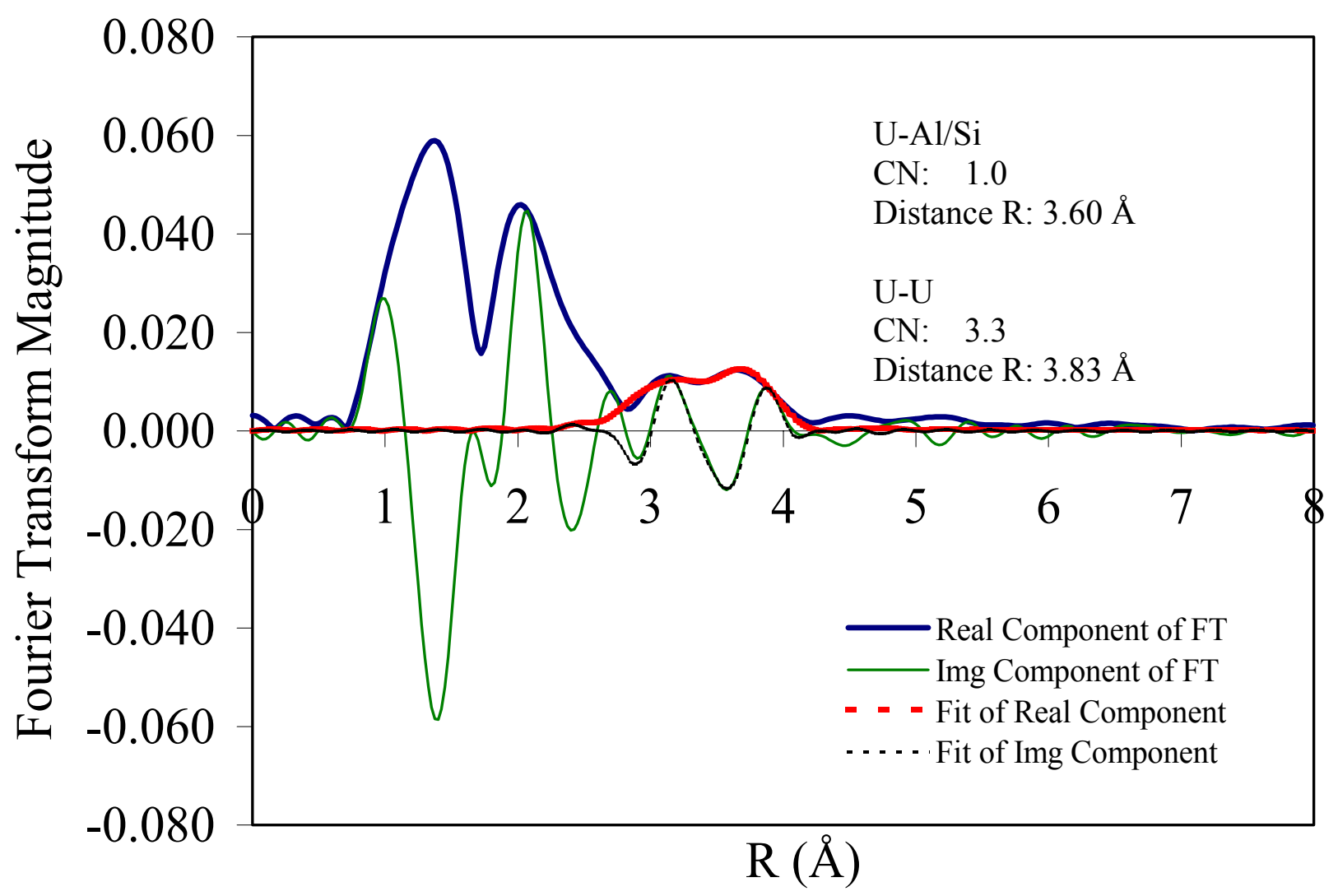

Figure 4.11 FT and second coordination shell fit data for the U reference sample (for the sodalite synthesis) - uncorrected for phase shift.] Although no Si was added to this sample, the best fits for a interaction at $3.6 \AA$ are made with Si. 


\subsubsection{Fourier-Tra nsformed Data for U in the Si-free U Reference Sample for the Zeolite A Synthesis}

The FT data and corresponding first shell fit data for the Si-free U reference solid (for the zeolite A synthesis) are shown in Fig. 4.12. The $\mathrm{U}$ in this solid has one set of uranyl-type axial $\mathrm{O}$ atoms at $1.84 \AA$ and six equatorial $\mathrm{O}$ atoms at $2.16 \AA$. These first coordination shell data differ from that obtained for the other two $\mathrm{U}$ reference solids as discussed in the previous sections.

Fits were performed for the second shell interactions as shown in Fig. 4.13. Fits for these outer shell interactions indicate the presence of one $\mathrm{Al}$ atom in the outer shell at $3.60 \AA$ (based on using $\mathrm{Si}$, which has a similar mass to that of $\mathrm{Al}$ ) in addition to three $\mathrm{U}$ atoms at $3.83 \AA$. These data suggest that a U(VI)-aluminate solid phase may exist in this sample. Additional work is recommended to examine the influence of $\mathrm{Al}$ on $\mathrm{U}$ solubility and the local coordination environment of $\mathrm{U}$ because $\mathrm{U}(\mathrm{VI})$-aluminate solids have not been documented. This U-XAFS study pertains to multiple sample forms of $\mathrm{U}$. Therefore, conclusions about higher shell behavior are less straightforward in this study than that which would be expected for studies with pure crystalline $\mathrm{U}$ solids (i.e., as for uraninite, $\mathrm{U}(\mathrm{IV}) \mathrm{O}_{2(\mathrm{~s})}$ ). 


\section{U Reference for Zeolite A}

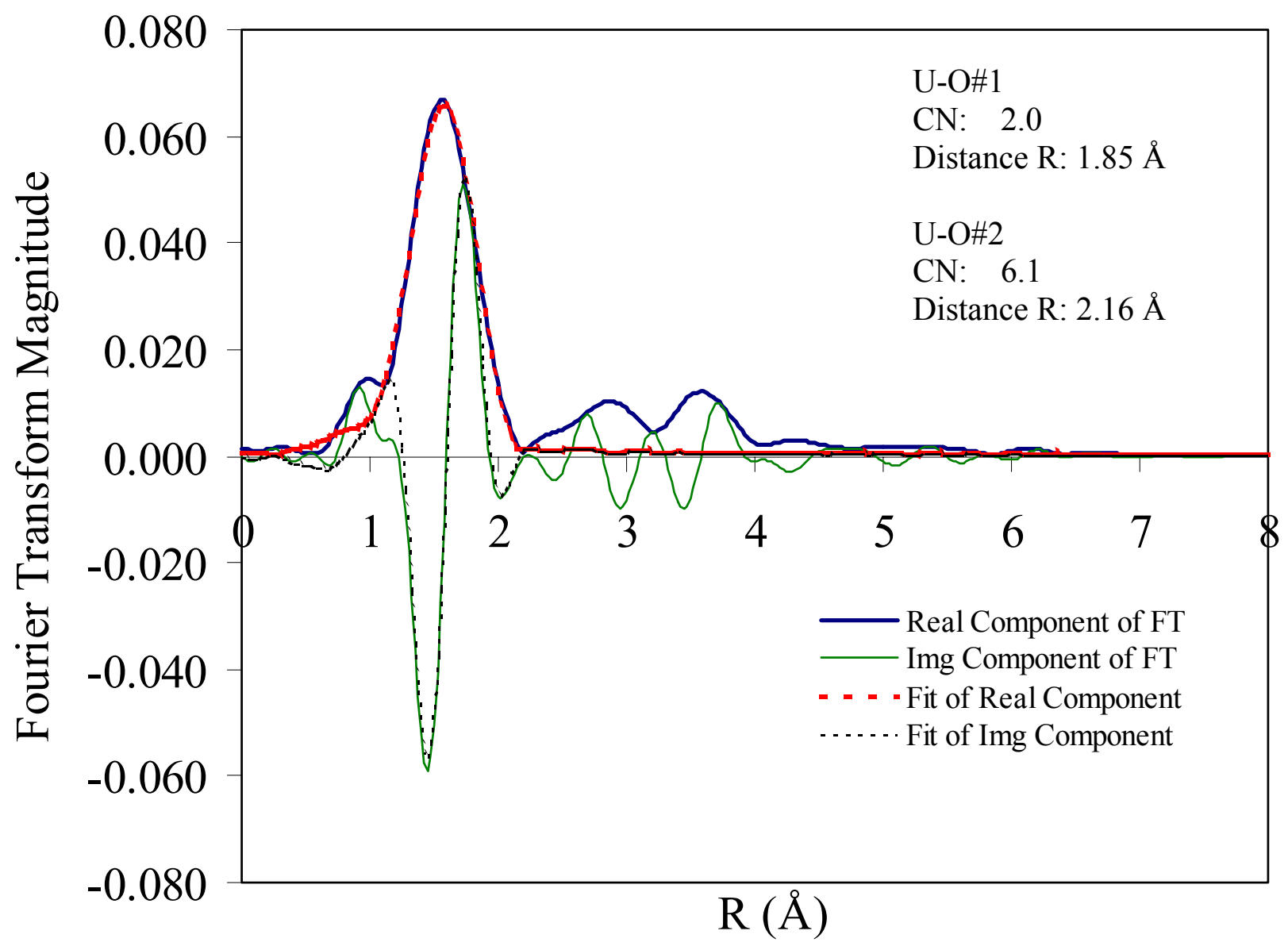

Figure 4.12 FT and first shell fit data for the U reference sample (for the zeolite A synthesis) uncorrected for phase shift. 


\section{U Reference for Zeolite A}

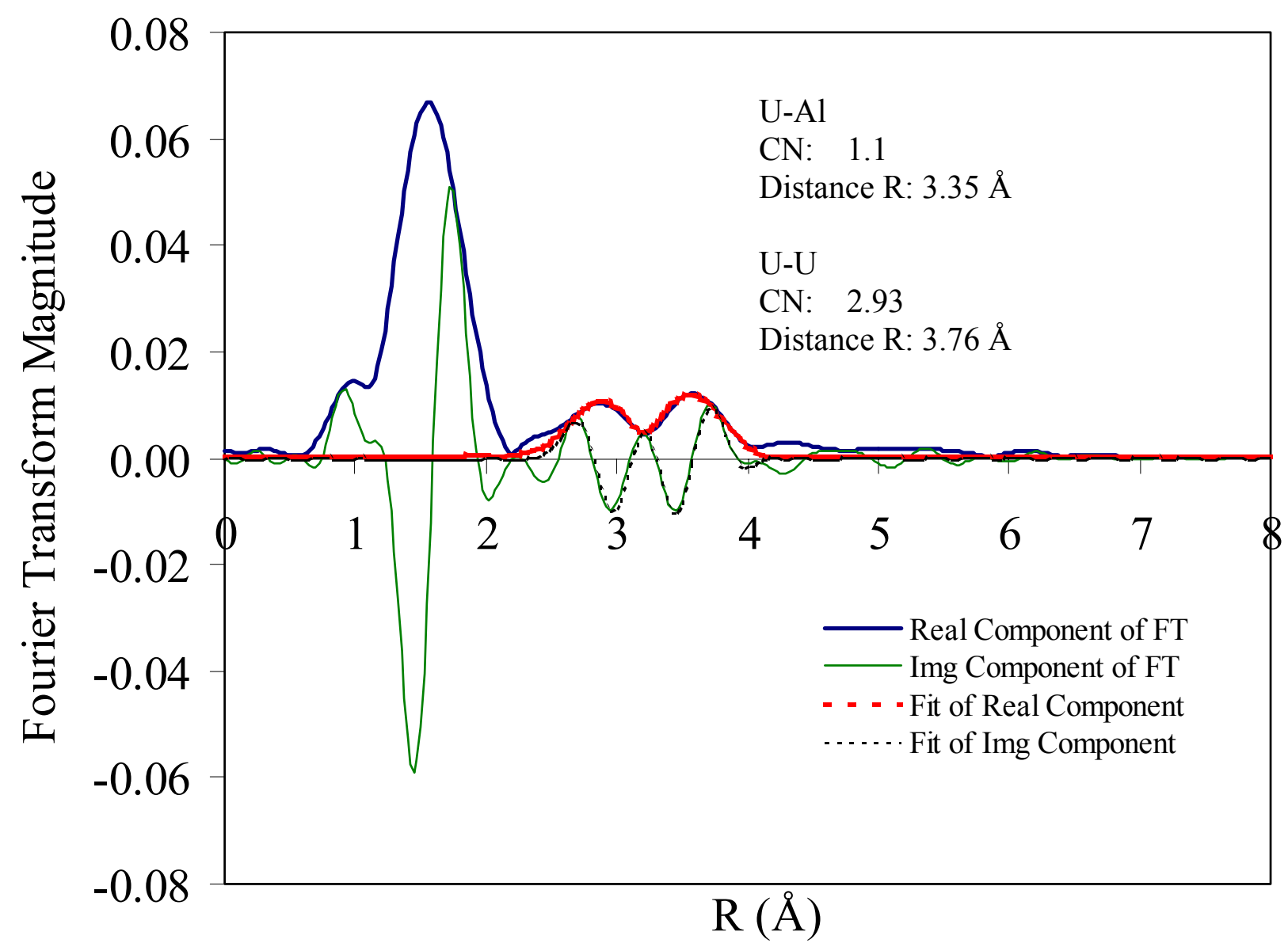

Figure 4.13 FT and outer shell fit data for the U reference sample (for the zeolite A synthesis) uncorrected for phase shift. 
WSRC-TR-2002-00510, REVISION 0

\subsubsection{Fourier-Transformed Data for U in Carbonate-washed Amorphous Zeolite}

The FT data and corresponding first and second shell fit data for the carbonate-washed amorphous zeolite sample are shown in Fig. 4.14. This solid has one set of uranyl-type axial O atoms at $1.79 \AA$ and three equatorial $\mathrm{O}$ atoms at $2.42 \AA$. A coordination number value of three for the equatorial $\mathrm{O}$ atoms is fairly low, based on typical literature values. Attempts to fit for a higher number of equatorial $\mathrm{O}$ atoms were unsuccessful. The FT data for the outer shells contains two Si atoms at a U-Si radial distance of $3.60 \AA \AA$ and three Si atoms at a U-Si radial distance of $3.92 \AA$. These long radial distances are indicative of Si corner sharing behavior with $\mathrm{U}$ polyhedra as previously discussed. Two outer shell $\mathrm{U}$ atoms were also identified to exist at $3.92 \AA$. Collectively, these data indicate that a uranyl silicate phase or phases are present in the carbonate-washed amorphous zeolite precursor sample. ${ }^{71}$

\subsubsection{Fourier-Tra nsformed Data for U in Carbonate-washed Amorphous Sodalite}

The FT data and corresponding first shell fit data for the carbonate-washed sodalite sample are shown in Fig. 4.15. The $\mathrm{U}$ in this solid has on average one set of three uranyl-type axial $\mathrm{O}$ atoms at $1.77 \AA$ and six equatorial $\mathrm{O}$ atoms at $2.47 \AA$. A value of three $\mathrm{O}$ atoms at a single axial U-O distance is one coordination number too high (two is physically ideal). These results do not indicate that there are two different $U$ phases. Our XAFS fits provide average values and if there were two sets of uranyl phases (specifically, axial $\mathrm{O}$ atoms at two different distances) we would expect to obtain a total coordination number value (i.e., sum) of 2 for these $\mathrm{O}$ atoms. More suitable fits for the first shell axial $\mathrm{O}$ atoms were not possible.

The FT data for the outer shells (Fig. 4.16) contains three Si atoms at a U-Si radial distance of $3.61 \AA$, which is a distance indicative of Si corner sharing behavior with U polyhedra as previously discussed. Three $\mathrm{U}$ atoms at a U-U radial distance of $3.86 \AA$ were also observed. Collectively, these data indicate that a uranyl silicate phase or phases are present in this carbonate-washed sodalite sample. These U-U model fit data are not representative of uranate phases, which have much longer U-U radial distances (e.g., $6 \AA$ for a cobalt uranate). ${ }^{60}$ 


\section{Carbonate Washed Amorphous Zeolite Precursor Material}

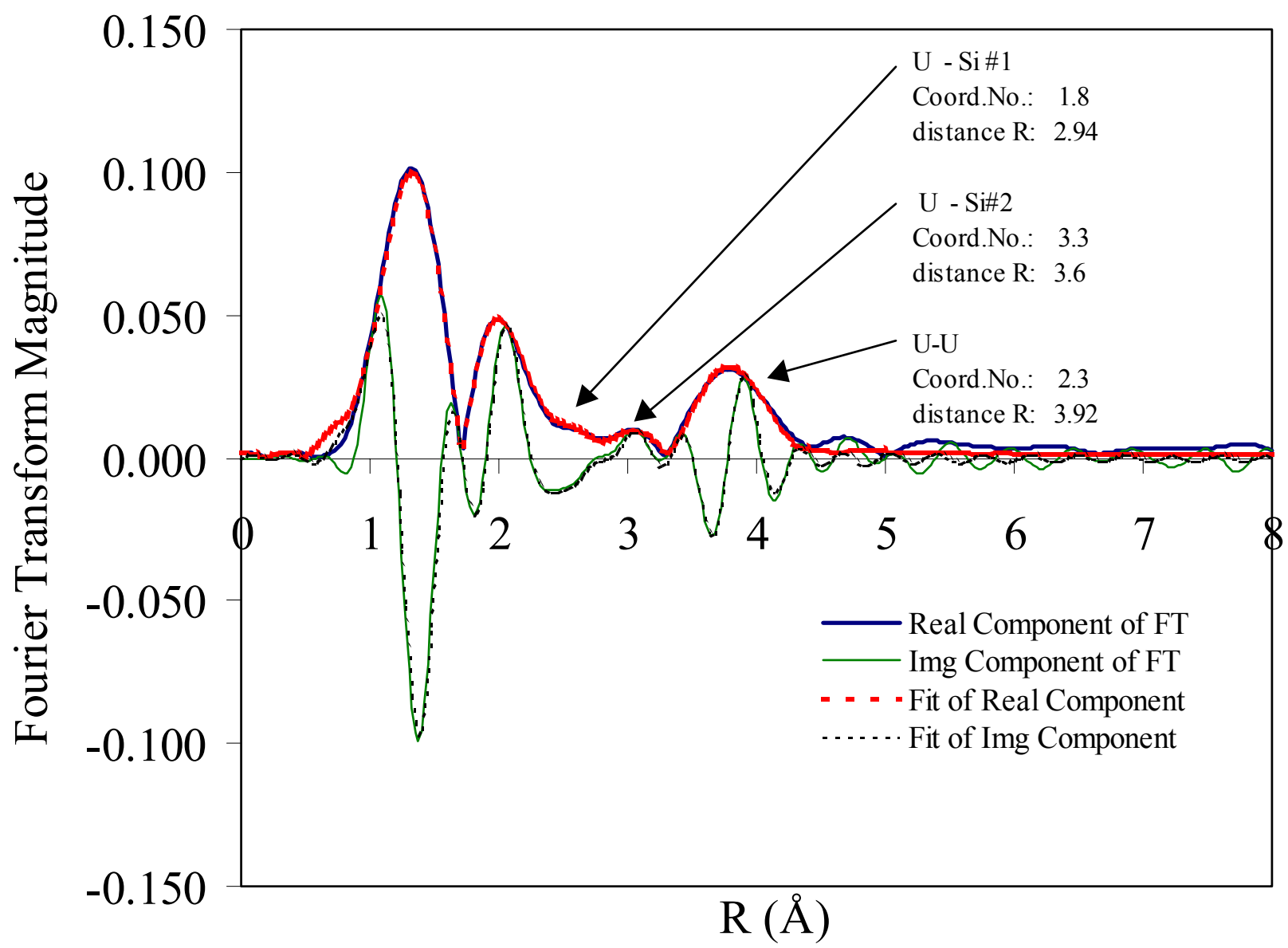

Figure 4.14 FT and multiple shell fit data for the carbonate-washed amorphous zeolite sampleuncorrected for phase shift. 


\section{Carbonate Washed Sodalite}

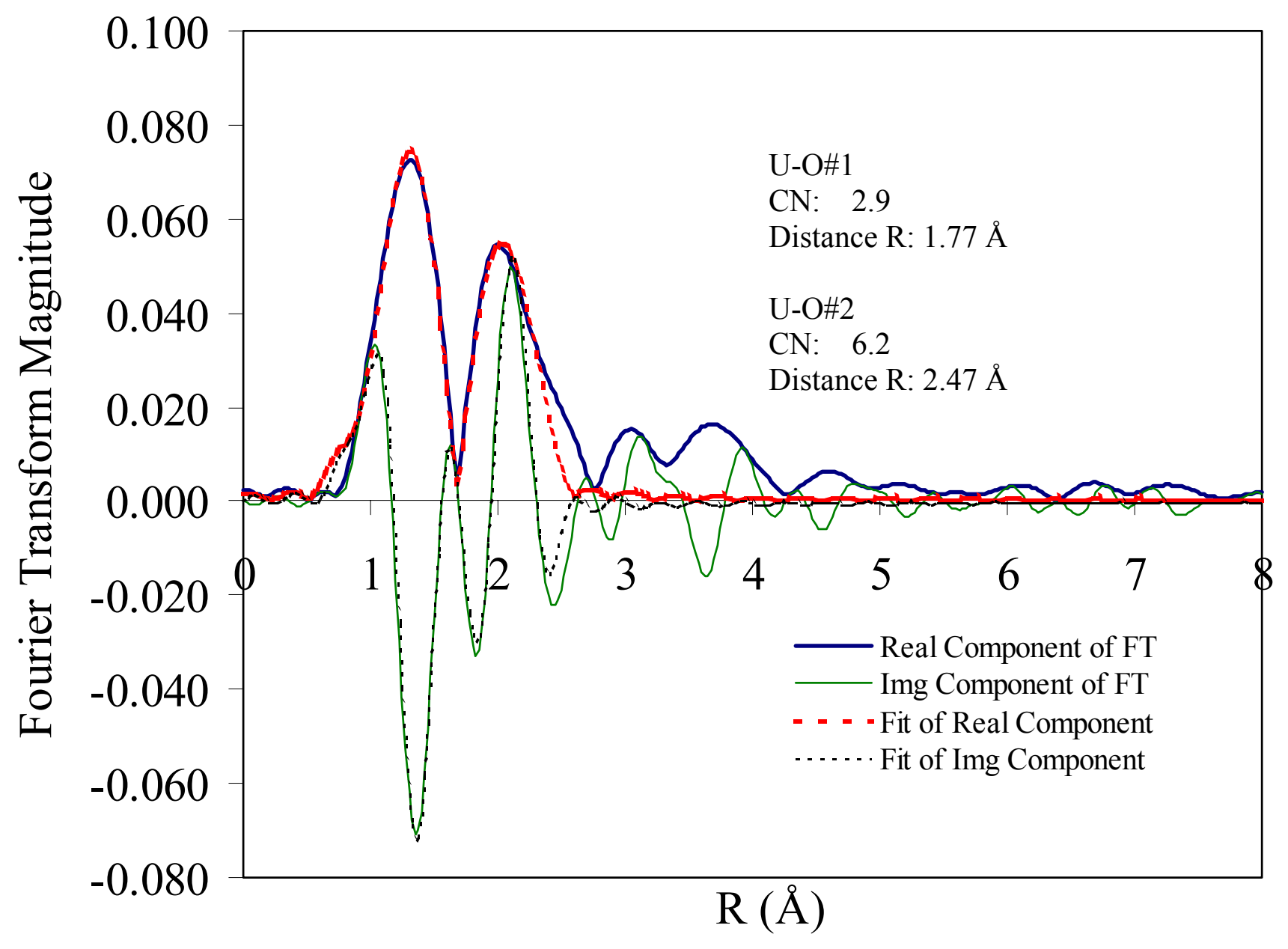

Figure 4.15 FT and first shell fit data for the carbonate-washed sodalite sample - uncorrected for phase shift. 


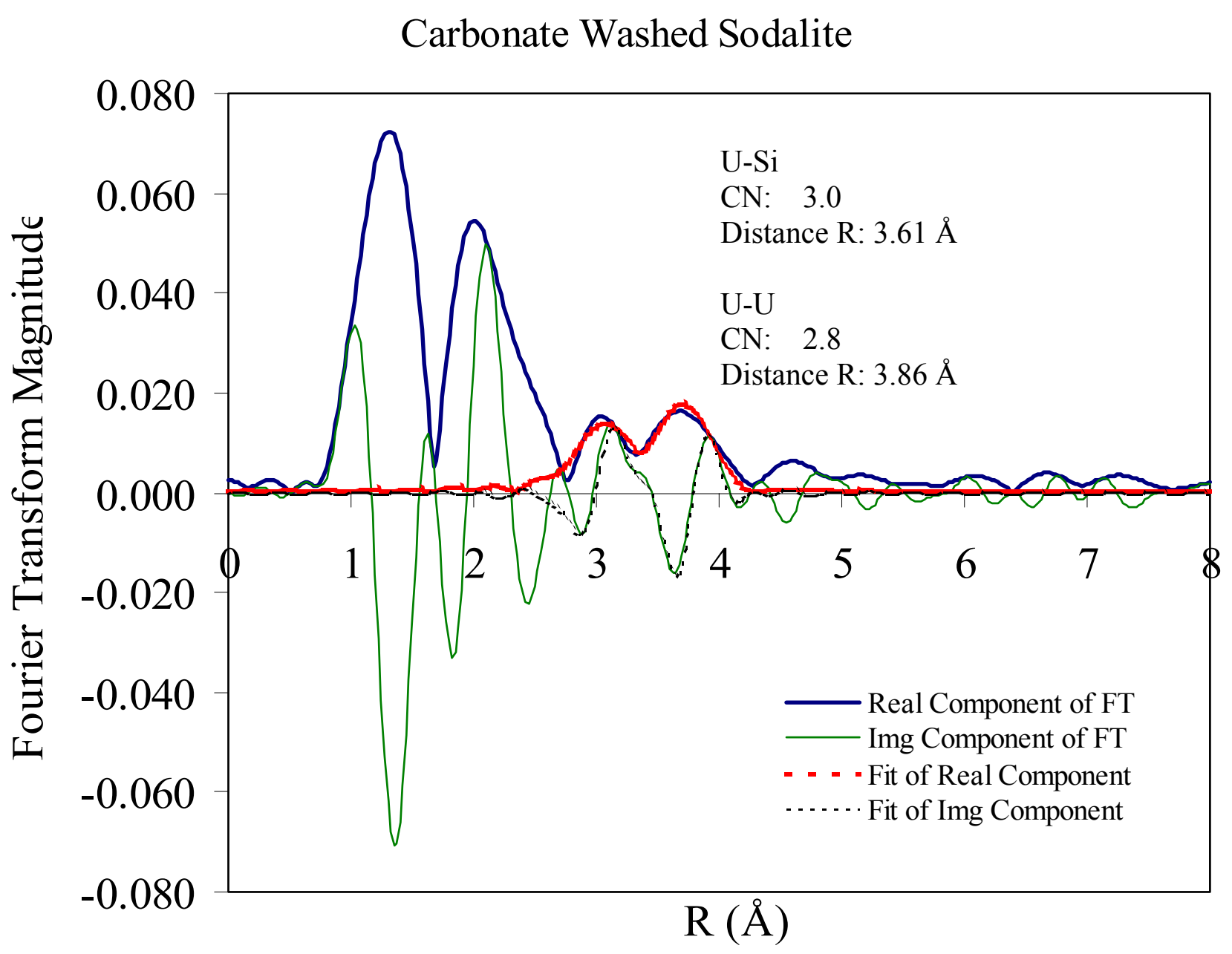

Figure 4.16 FT and outer shell fit data for the carbonate-washed sodalite sample-uncorrected for phase shift. 


\subsubsection{Influence of Multiple Local Coordination Environments on the U-XAFS Data Analyses}

XAFS techniques are typically best used when one or two populations of species exist in a sample of interest. In our case, we observe more than one population of solid U phases.

However, for $\mathrm{U}$ solid phases, resolution of XAFS spectra can be more difficult. For example, the structures of some $\mathrm{U}$ solids like schoepite $\left[\mathrm{UO}_{2}(\mathrm{OH})_{2} \cdot 2 \mathrm{H}_{2} \mathrm{O}\right]$ permit the $\mathrm{U}$ to exist in up to eight different radial atom positions (i.e., there are eight different unique environments for $\mathrm{U}$ in schoepite). Because XAFS data are representative of the average local environment, the eight UU distances (ranging between 3 and $4 \AA$ ) that exist with this solid may be difficult to resolve in the FT data due to constructive and destructive interference between the oscillations associated with the eight different U-U interactions. The presence of multiple environments results in several overlapping shells, which creates some difficulties when coordination numbers (CN's) and to a somewhat lesser degree, bond distances are sought during model fit analyses. 
WSRC-TR-2002-00510, REVISION 0

\subsection{DISCUSSI ON AND CONCLUSIONS}

\subsection{Discussion of the XAFS Data from the Si-free U Reference Solids.}

The XAFS data for the U phases from the Si-free U reference syntheses indicate that uranyl-type and uranate-type solids are formed. The presence of outer shell $U$ in the FT data for two of the samples suggests that the $\mathrm{U}$ precipitated during synthesis. These solids appear to contain $\mathrm{Al}-$ although no quantitative analyses for Al were performed. A more thorough verification of this would require more analyses and syntheses that are aimed at the study of $U$ solids formation in Al-rich caustic solutions. The presence of multiple environments for the $U$ in these three samples limited our ability to make additional interpretations of the data.

The U-XAFS data for the precipitated solids from the $U$ reference for the sodalite and zeolite A synthesis materials indicate that bonding with $\mathrm{Al}$ (in the outer coordination shells) may have occurred. It is also possible that bonding with "tramp" Si (coming from the reagent chemicals used to make the salt solutions) rather than Al may have also occurred. The U-XAFS data for the $\mathrm{Si}$-free $\mathrm{U}$ reference solid from the amorphous zeolite precursor preparation did not indicate the presence of Al bonding in the outer coordination shells. The absence of Al interactions may be due to the lower temperature (40 degrees C) used during these syntheses as opposed to the higher temperatures ( 80 and 90 degrees $C$ ) that were used for the other syntheses (i.e., those for the reference $U$ solids that were part of the sodalite and zeolite A syntheses).

\subsection{Discussion of the XAFS Data from the Unwashed, DI-washed and Carbonate-washed NAS Solids.}

The XAFS data for the unwashed and DI-washed NAS solids showed evidence of outer coordination shell $\mathrm{O}, \mathrm{U}$ and $\mathrm{Si}$ (data note shown) - suggesting that uranyl oxyhydroxide and uranyl silicate solids exist in these samples. However, we could not resolve CN or radial distance information from the recorded spectra due to the presence of multiple local $U$ environment in these samples limited our ability to make more definitive interpretations of the data.

The XAFs data for the carbonate-washed samples exhibited characteristics that are indicative of fewer local U environments (i.e., possibly, a single U(VI) phase) as opposed to three or more as was probably present in the unwashed and DI-washed samples. The model fit data for the carbonate-washed sodalite and amorphous zeolite samples indicates that a uranyl-like silicate phase is also present in the samples. This phase has similar features to the uranyl silicate solids that are shown in Figure 4.2. This is because the U-Si radial distances are indicative of mostly corner sharing U(VI) with $\mathrm{SiO}_{4}$ tetrahedra (radial distances greater than $3.2 \AA$ ) and some edge sharing $\mathrm{U}$ with $\mathrm{SiO}_{4}$ tetrahedra (radial distances less than $3 \AA$ ). The absence of significant edge sharing behavior supports the formation of uranyl silicates as opposed to sorbed U(VI) species on NAS because sorbed U typically attaches to aluminosilicate and silica-containing solids via edge linking as shown in Table 4-1 and as previously discussed in Section 4.1. The number of $\mathrm{U}-\mathrm{U}$ interactions in the carbonate-washed NAS samples suggests the precipitation of a better 
WSRC-TR-2002-00510, REVISION 0

defined uranyl silicate phase (such as Na-weeksite and Na-boltwoodite) occurred during the UNAS syntheses.

The precipitation of uranyl silicate and hydroxide phases could have occurred on the NAS surface or without the NAS surfaces. The XAFS data could not determine the nature of the precipitation (i.e., whether it was on NAS solids or not). However, the formation of a large amount of NAS solids relative to the low amount of $U$ in the NAS solids could help seed the precipitation of $U$ solids. Uptake of the $U$ into the sodalite structure was possible, however, the uranyl silicate particles (which contain on average, several U atoms) that precipitated are clearly too large in size to exist in the sodalite structural cages or within the zeolite A tunnels. We estimate that the particle size (on average) of the precipitate material was colloidal in nature but too large to fit within the sodalite and zeolite frameworks. However, our XAFS data represent average radial distance and coordination number information. It is possible that small monomeric species may be exist within the zeolite structures in the presence of larger $U$ polymeric species.

Because this phase remains in the samples after leaching with DI water and carbonate, it is resistant to leaching. This stable (i.e., sparingly soluble) residual phase is most likely colloidal in nature and not likely to be detected by XRD analyses because of its short-range order. If formed in the $2 \mathrm{H}$ evaporator, a uranyl silicate-type phase may convert to sodium diuranate over time or may have not been detected.

Studies show that in the environment, the uranyl silicate solids are highly stable mineral phases in that they dissolve more slowly than other mineral phases under similar conditions. Uranophane $\left[\mathrm{Ca}\left(\mathrm{H}_{3} \mathrm{O}\right)_{2}\left(\mathrm{UO}_{2}\right)_{2}\left(\mathrm{SiO}_{4}\right)_{2} \cdot 3 \mathrm{H}_{2} \mathrm{O}\right]$ in particular has been identified as the most common U(VI) mineral in the environment, which suggests this mineral is fairly resistant to weathering in natural waters. ${ }^{72}$ Dissolution studies have been done with uranyl silicates (uranophane and soddyite) in the presence of carbonate ion. ${ }^{73,74,75}$ However, only one study has examined the effect of carbonate concentration on uranyl silicate dissolution rates. ${ }^{74}$ This study found that carbonate ion increases the rate of soddyite dissolution. Another study examined the dissolution of schoepite and soddyite in $0.5 \mathrm{M} \mathrm{NaCO}_{3}$ in batch. This study showed that schoepite dissolved 2.5 times faster than soddyite. The higher dissolution rate is thought to be due to the simplicity of the schoepite structure--relative to that of soddyite. For example, the $\mathrm{U}(\mathrm{VI})$ minerals with $\mathrm{SiO}_{4}$ groups contain repeating units or "sheets" of tetrahedral group linkages whereas schoepite does not contain these linkages. ${ }^{72}$ These silica tetrahedral linkages could be less prone to dissolution and therefore influence mineral dissolution rates. Hence, $\mathrm{U}(\mathrm{VI})$ solids such as schoepite, which do not have $\mathrm{SiO}_{4}$ (silica tetrahedra) in their structures, are likely to be more soluble than minerals that have silica tetrahedra in their structures.

Additionally, many of our fits resulted in the generation of local first shell coordination numbers that were inconsistent with that for known solid U species - such as the presence of two U-O axial distances and the low numbers of equatorial U-O coordination numbers (as noted in Fig. 4.6). Again, there are complexities associated with the multiple local environments. However, the radial distance information is more reliable than that of the $\mathrm{CN}$ values because for XAFS analyses, radial distance information is more readily obtained than that of $\mathrm{CN}$ information. 
In summary, the presence of $\mathrm{Al}$ and $\mathrm{Si}, \mathrm{U}$ will precipitate during NAS formation as uranyl silicate and uranyl oxyhydroxide (schoepite-like) phases, which have a strongly amorphous behavior. Future related studies could be conducted to characterize $U$ solids that will form in high Si and Al caustic salt solutions or U-NAS solids that form during the precipitation of the zeolite called cancrinite.

\subsection{QUALITY ASSURANCE}

The following documents govern the work reported in this document.

- M. C. Duff, D. B. Hunter and B. Croy, "Task Technical and Quality Assurance Plan for Task technical for XAFS Analyses on Solids from Sorption and Co-precipitation Studies with Uranium Sodium Aluminosilicates," WSRC-RP-2002-00021, Rev. 1, February 13, 2002.

- Notebook WSRC-NB-2001-00052 (M. C. Duff) contains the experimental XAFS data obtained from this work.

\subsection{ACKNOW LEDGEMENTS}

The authors acknowledge J. Woicik (National Institutes of Standards and Technology), A. Ackerman (BNL), M. McAvoy (BNL), R. Zantopp (BNL), W. Rao (University of Georgia), and T. Lanzirotti (University of Chicago) for their help on this project. The authors thank R. Edwards (WSRC), S. Reboul (WSRC), P. Jackson (DOE), B. Van-Pelt (SRTC) and W. Tamosaitis (SRTC) for their assistance, support and ideas. Mrs. B. Croy (SRTC) and A. L. Williams (SRTC) provided excellent laboratory assistance with sample preparation. Mrs. C. Pierce (WSRC) provided excellent health physics support in the laboratory. Drs. W. R. Wilmarth, S. Rosencrance and D. T. Hobbs (all of SRTC) provided many ideas associated with this work and related efforts to better understand the interaction of U with NAS solids. 
WSRC-TR-2002-00510, REVISION 0

\subsection{REFEREN CES}

\footnotetext{
${ }^{1}$ Stumm, W. (1992). Chemistry of the Solid-Water Interface: Processes at the Mineral-Water and Particle-Water Interface in Natural Systems. Wiley-Interscience, NY.

${ }^{2}$ McBride, M. B. (1994). Environmental Chemistry of Soils. Oxford Press, NY.

${ }^{3}$ Due to the small nature of nanoparticles, they often have more atoms at the solid-water interface than inside the solid. The use of spectroscopic techniques to elucidate the exact location of sorbing metals in such solids may potentially result in an inability to determine whether the metal is in an interlayer or vacancy site.
}

${ }^{4}$ Sorption reversibility is tested by leaching the metal loaded solid with a target metal-free solution that has the same properties (i.e. ionic strength, background electrolytes, $\mathrm{pH}$ etc...) and measuring the leachate solution to determine whether any target metal is released. Specifically adsorbed species are typically not leached with lower ionic strength solutions and specific adsorption processes are not a function ionic strength (assuming the electrolytes do not interact with the sorbed species via complexation etc.). Outersphere species can be readily removed with solutions of low ionic strength or solutions that contain high concentrations of a cation that has a higher affinity for the surface than the outer-sphere species.

Therefore, outer-sphere sorption is viewed as reversible and a function of ionic strength.

${ }^{5}$ Specific adsorption is typically a function of surface charge properties. When a participating surface has a charge that varies with solution $\mathrm{pH}$, specific adsorption exhibits $\mathrm{pH}$-dependent behavior. For example, a surface that is highly protonated (at low $\mathrm{pH}$ ) will have more affinity for negatively-charged species (such as the negatively-charged species). As the $\mathrm{pH}$ decreases, the surface charge of $\mathrm{pH}$-dependent charged surfaces becomes more negative and the uptake of positively charged species is favored. Therefore, the surface of metal oxides for example specifically adsorbs more positively charged species as the solution $\mathrm{pH}$ increases. However, this behavior is generalized. As the solution $\mathrm{pH}$ increases sorbing species undergoes a change in speciation - such as hydrolysis. In this case, the amount of specific adsorption becomes a function of solution speciation. Ion exchange resins that sorb free ions of actinides (e.g., the non-hydrolyzed $\mathrm{Pu}^{4+}$ species) at high $\mathrm{H}^{+}$concentrations (i.e., low $\mathrm{pH}$ ) will release sorbed $\mathrm{Pu}^{4+}$ species when the solution $\mathrm{pH}$ is raised-due to the hydrolysis of the sorbed $\mathrm{Pu}^{4+}$ (e.g., $\mathrm{Pu}(\mathrm{OH})_{3}{ }^{+}$, $\mathrm{Pu}(\mathrm{OH})_{2}{ }^{2+}$ ). The hydrolysis species have a lower overall charge and their size (as an ion group) is larger than the free ion-making them have a lower affinity for the surface. Other processes [particularly for $\mathrm{U}(\mathrm{VI})]$ that can influence sorption are metal complexation with ligands (i.e., with carbonate ion) or in the case of anions, protonation (e.g., $\mathrm{PO}_{4}{ }^{3-}+\mathrm{H}^{+} \rightarrow \mathrm{HPO}_{4}{ }^{2-}$ ). Specific adsorption processes are a function of many variables.

${ }^{6}$ Olguin, M. T., Duque, J., Pomés, R., Villafuerte-Castrejón, M. E., Sansores, L. E., Bosch, P. and Bulbulian, S. (1997). Characterization of $\mathrm{UO}_{2}{ }^{2+}$-exchanged Y zeolite. J. Radio. Anal. Nucl. Chem. 222, 235-237.

${ }^{7}$ No U-aluminate solid phases have been documented. If these phases form, other U phases, such as the oxides, hydroxides and silicates of $U$ are more likely dominate the speciation of solid phase $U$ under evaporator and salt solution conditions.

${ }^{8}$ Li, Y. and Burns, P. C. (2001). The structures of two sodium uranyl compounds relevant to nuclear waste disposal. J. Nucl. Mater. 299, 219-226. 
${ }^{9}$ Hobbs, D. T., Bronikowski, M. G., Edwards, T. B. and Pulmano, R. L. (1999). Final Report on Phase III Testing of Monosodium Titanate Adsorption. WSRC-TR-99-00134, May 28, 1999.

${ }^{10}$ Duff, M. C., Hobbs, D. T. and Fink, S. D. (2002). "Permanganate Treatment Optimization Studies for Strontium and Actinide Removal from High Level Waste Simulants," WSRC-TR-2002-0027, Jan. 14, 2002.

${ }^{11}$ Crespo, M. T., Gascon, J. L. and Acena, M. L. (1993). Techniques and analytical methods in the determination of uranium, thorium, plutonium, americium and radium by adsorption on manganese dioxide. Sci.Tot.Environ. 130, 383-391.

${ }^{12}$ Duff, M. C., Hunter, D. B., Hobbs, D. T., Jurgensen, A. and Fink, S. D. (2002). "Characterization of Sorbed Plutonium, Neptunium, Strontium on Manganese Solids from Permanganate Reduction." WSRCTR-2002-00366, Rev. 0, Sept. 19, 2002.

${ }^{13}$ Clark, D. L., Conradson, S. A., Donohoe, R. J., Keogh, D. W., Morris, D. E., Palmer, P. D., Rogers, R. D. and Tait, C. D. (1999). Chemical speciation of the uranyl ion under highly alkaline conditions, synthesis, structures and oxo ligand exchange dynamics. Inorg. Chem. 38, 1456-1466.

${ }^{14}$ Hsi, C-K. D. and Langmuir, D. (1985). Adsorption of uranyl onto ferric oxyhydroxides: Applications of the surface complexation site-binding model. Geochim. Cosmochim. Acta 49, 1931-1941.

${ }^{15}$ Duff, M. C. and Amrhein, C. (1996). Uranium(VI) adsorption on goethite and soil in carbonate solutions. Soil Sci. Soc. Amer. J. 743, 1393-1400.

${ }^{16}$ Duff, M. C., Hunter, D. B., Hobbs, D. T., Barnes, M. J. and Fink, S. D. (2001). "Characterization of Sorbed Uranium, Plutonium and Neptunium on Monosodium Titanate," WSRC-TR-2001-00356, Sept. 19, 2001.

${ }^{17}$ Akyıl, S., Aslani, M. A. A., Ǒlmez, Ş. and Eral, M. (1996). Kinetic studies of uranium(VI) adsorption on a composit ion exchanger. J. Radioanal. Nucl. Chem. Lett. 213, 441-450.

${ }^{18}$ El Aamrani, F. Z., Duro, L., De Pablo, J. and Bruno, J. (2002). Experimental study and modeling of the sorption of uranium(VI) onto olivine rock. Appl. Geochem. 17, 399-408.

${ }^{19}$ Akyıl, S., Aslani, M. A. A. and Aytaş, Ş. (1998). Distribution of uranium on zeolite X and investigation of thermodynamic parameters for this system. J. Alloys Cpds 271/273, 769-773.

${ }^{20}$ Prikryl, J. D., Jain, A., Turner, D. R. and Pabalan, R. T. (2001). Uranium(VI) sorption behavior on silicate mineral mixtures. J. Contam. Hydrol. 47, 241-253.

${ }^{21}$ Reich, T., Moll, H., Denecke, M. A., Geipel, G. Bernhard, G., Nitsche, H., Allen, P. G., Buchner, J. J., Kaltsoyannis, N., Edlestein, N. M. and Shuh, D. K. (1996). Characterization of hydrous uranyl silicate by EXAFS. Radiochim. Acta 74, 219-223.

${ }^{22}$ Duff, M. C., Coughlin, J. U. and Hunter, D. B. (2002). Uranium co-precipitation with Fe oxide minerals, Geochim. Cosmochim. Acta 66, 3533-3547. 
${ }^{23}$ Bargar, J. R., Reitmeyer, R., Lehnart, J. J. and Davis, J. A. (2000). Characterization of U(VI)-carbonato ternary complexes on hematite: EXAFS and electrophoretic mobility measurements. Geochim.

Cosmochim. Acta. 64, 2737-2749.

${ }^{24}$ Olguin, M. T., Duque, J., Pomes, R., Villafuerte-Castrejon, M. E., Sansores, L. E., Bosch, P. and Bulbulian, S. (1997). Characterization of $\mathrm{UO}_{2}{ }^{2+}$-exchanged Y zeolite. J. Radioanal. Nucl. Chem. 222, 235-237.

${ }^{25}$ Azenha, M. E. D. G., da Graça Miguel, M., Formosinho, S. J. and Burrows, H. D. (2001). The characterization by luminescence spectroscopy of uranium(VI) incorporated into zeolites and aluminas. $J$. Molec. Structure 563-564, 439-442.

${ }^{26}$ Koningsberger, D. C. and Prins, R. (1988). X-ray Absorption: Techniques of EXAFS, SEXAFS and $X A N E S$, Wiley, New York.

${ }^{27}$ Stern, E. A. (1974). Theory of extended X-ray absorption fine structure. Phys. Rev. B10, 3027-3037.

${ }^{28}$ Lieser, K. H., Quandt-Klenk, S. and Thybusch, B. (1992). Sorption of uranyl ions on hydrous silicon dioxide. Radiochim. Acta 57, 45-50.

${ }^{29}$ Mensah, A., Li, J. and Zbik, M. (2002). "The Chemistry, Crystallization, Physicochemical Properties and Behavior of Sodium Aluminosilicate Solid Phases," WSRC/ERDA GA00083. June 2002.

${ }^{30}$ Oji, L. N. and Williams, A. L. (2002). "Evaluation of the Incorporation of Uranium into Sodium Aluminosilicate Phases (U)," WSRC-2002-TR-00527, Rev. 0, November, 2002

${ }^{31}$ Ressler, T. (1999). WinXAS. A program for X-ray absorption spectroscopy data analysis under MS Windows. J. Synchr. Rad. 5, 118-122.

${ }^{32}$ Newville, M., Livins, P., Yacoby, Y., Rehr, J. J. and Stern, E. A. (1993). Near-edge X-ray-absorption fine-structure of $\mathrm{Pb}$ - A comparison of theory and experiment. Phys. Rev. B-Cond. Matter, 47, 1412614131.

${ }^{33}$ Sayers, D. E. and Bunker, B. A. (1988). In X-ray Absorption: Techniques of EXAFS, SEXAFS and $X A N E S$. Koningsberger, D. C. and Prins, R. (eds). Wiley, New York, Chap. 6.

${ }^{34}$ FEFF is a software package that was generated by researchers at the Univ. of Washington with DOE funding support. Access and use of this software is free within the DOE complex.

${ }^{35}$ Mustre de Leon, J., Rehr, J. J., Zabinsky, S. I. and Albers, R. C. (1991). Ab initio curved-wave x-rayabsorption fine structure, Phys. Rev. B44, 4146.

${ }^{36}$ Rehr, J. J. and Albers, R. C. (1990). Scattering-matrix formulation of curved-wave multiple-scattering theory: Application to x-ray-absorption fine structure. Phys. Rev. B41, 8139.

${ }^{37}$ Rehr, J. J., Mustre de Leon, J., Zabinsky, S. I. and Albers, R. C. (1991). Theoretical X-ray absorption fine structure standards. J. Am. Chem. Soc. 113, 5135.

${ }^{38}$ Rehr, J. J., Zabinsky, S. I. and Albers, R. C. (1992). High-order multiple scattering calculations of xray-absorption fine structure. Phys. Rev. Let. 69, 3397. 
${ }^{39}$ Stern, E. A., Newville, M., Ravel, B., Yacoby, Y. and Haskel, D. (1995). The UWAFS analysis package - Philosophy and details. Physica B. 208-209, 117-120.

${ }^{40}$ Moyes, L. N., Parkman, R. H., Charnock, J. M., Vaughan, D. J., Livens, F. R., Hughes, C. R. and Braithwaite, A. (2000). Uranium uptake from aqueous solution by interaction with goethite, lepidocrocite, muscovite, and mackinawite: An X-ray absorption spectroscopy study. Environ. Sci. Technol. 34, 10621068.

${ }^{41}$ Hudson, E. A., Terminello, L. J., Viani, B. E., Denecke, M., Reich, T., Allen, P. G., Bucher, J. J., Shuh, D. K. and Edelstein, N. M. (1999). The structure of $\mathrm{U}^{6+}$ sorption complexes on vermiculite and hydrobiotite. Clays Clay Min. 47, 439-457.

${ }^{42}$ Chisholm-Brause, C., Conradson, S. D., Buscher, C. T., Eller, P. G. and Morris, D. E. (1994). Speciation of uranyl sorbed at multiple binding sites on montmorillionite. Geochim. Cosmochim. Acta. 58, 3625-3631.

${ }^{43}$ Waite, T. D., Davis, J. A., Payne, T. E., Waychunas, G. A. and Xu, N. (1994). Uranium adsorption to ferrihydrate: Application of a surface complexation model. Geochim. Cosmochim. Acta. 58, 5465-5478.

${ }^{44}$ Wersin, P., Hochella, M. F., Persson, J., Redden, G., Leckie, J. O. and Harris, D. W. (1994). Interaction between aqueous uranium(VI) and sulfide minerals: Spectroscopic evidence for sorption and reduction. Geochim. Cosmochim. Acta 58, 2829-2843.

${ }^{45}$ Bargar, J. R., Reitmeyer, R. and Davis, J. A. (1999). Spectroscopic confirmation of uranium(VI)carbonato adsorption complexes on hematite. Env. Sci. Technol. 33, 2481-2484.

${ }^{46}$ Sylwester, E. R., Hudson, E. A. and Allen, P. G. (2000). The structure of uranium(VI) sorption complexes on silica, alumina and montmorillonite. Geochim. Cosmochim. Acta. 64, 2431-2438.

${ }^{47}$ Lieser, K. H., Quandtklenk, S. and Thybusch, B. (1992). Sorption of uranyl ions on hydrous silicon dioxide. Radiochim. Acta 57, 45-50.

${ }^{48}$ Bargar, J. R., Reitmeyer, R., Lehnart, J. J. and Davis, J. A. (2000). Characterization of U(VI)-carbonato ternary complexes on hematite: EXAFS and electrophoretic mobility measurements. Geochim. Cosmochim. Acta. 64, 2737-2749.

${ }^{49}$ Allen, P. G., Shuh, D. K., Bucher, J. J., Edelstein, N. M., Palmer, P. A., Silva, R. J., Nguyen, S. N., Marquez, L. N. and Hudson, E. A. (1996). Determinations of uranium structures by EXAFS: Schoepite and other U(VI) oxide precipitates. Radiochim. Acta 75, 47-53.

${ }^{50}$ Thompson, H. A., Brown, G. E. and Parks, G. A. (1997). XAFS spectroscopic study of uranyl coordination in solids and aqueous solution. Amer. Mineral. 82, 483-496.

${ }^{51}$ Allard, T., Ildefonse, P., Beaucaire, C. and Calas, G. (1999). Structural chemistry of uranium associated with $\mathrm{Si}, \mathrm{Al}, \mathrm{Fe}$ gels in a granitic uranium mine. Chem. Geol. 158, 81-103.

${ }^{52}$ Sturchio, N. C., Antonio, M. R., Soderholm, L., Sutton, S. R. and Brannon, J. C. (1998). Tetravalent uranium in calcite. Science 281, 971-973. 
${ }^{53}$ Reeder, R. J., Nugent, M., Tait, C. D. and Morris, D. E. (2000). Uranyl incorporation into calcite and aragonite: XAFS and luminescence studies. Environ. Sci Technol. 34, 638-644.

${ }^{54}$ Smectites are phylloaluminosilicates mineral consisting of layers of aluminum octahedra and silicon tetrahedra that contain metals (such as $\mathrm{Fe}^{2+, 3+} \mathrm{Mn}^{2+}$ and $\mathrm{Mg}^{2+}$ ) within the structural vacancies. Fixed charge sites on aluminosilicate clays do not exhibit $\mathrm{pH}$-dependent surface charge behavior. These fixed charge sites typically sorb solution ions via an outer sphere/electrostatic mechanism. The fixed charge of the sites comes from an imbalance in internal structural charge due to the substitution of metal ions with a different charge than that which would normally fulfill charge balance in the clay structure (specifically, $\mathrm{Al}^{3+}$ and $\mathrm{Si}^{4+}$ ). On the edges of these structural layers are broken edge sites, which exhibit $\mathrm{pH}$-dependent charge behavior due to their propensity to protonation and deproton-ation. It is at these sites that specific adsorption of metals such as uranyl can occur.

${ }^{55}$ Turner, G. D., Zacahara, J. M., McKinley, J. P. and Smith, S. C. (1996). Surface-charge properties and $\mathrm{UO}_{2}{ }^{2+}$ adsorption of a subsurface smectite. Geochim. Cosmochim. Acta. 60, 3399-3414.

${ }^{56}$ Zachara, J. M. and McKinley, J. P. (1993). Influence of hydrolysis on the sorption of metal cations by smectites: Importance of edge coordination reactions. Aquatic Sciences. 55, 250-261.

${ }^{57} \mathrm{Li}$, Y. and Burns, P. C. (2001). The structures of two sodium uranyl compounds relevant to nuclear waste disposal. J. Nucl. Mater. 299, 219-226.

${ }^{58}$ Burns, P. C. (1999). The Crystal Chemistry of Uranium. In: Uranium: Mineralogy, Geochemistry and the Environment. Reviews in Mineralogy 38, pp. 23-90, Mineral. Soc. Amer.

${ }^{59}$ Burns, P. C., Ewing, R. C. and Hawthorn, F. C. (1997). The crystal chemistry of hexavalent uranium: Polyhedral geometries, bond-valence parameters, and polymerization of polyhedra. Can. Mineral. 35, 1551-1570.

${ }^{60}$ Clark, D. L., Conradson, S. A., Donohoe, R. J., Keogh, D. W., Morris, D. E., Palmer, P. D., Rogers, R. D. and Tait, C. D. (1999). Chemical speciation of the uranyl ion under highly alkaline conditions, synthesis, structures and oxo ligand exchange dynamics. Inorg. Chem. 38, 1456-1466.

${ }^{61}$ Duff, M. C., Hunter, D. B., Barnes, M. J. and Fink, S. D. (2002). "Characterization of uranium and mercury speciation in high level waste tank 8F and 11H sludge," WSRC-TR-2001-00428, Rev. 0.

${ }^{62}$ Reich, T., Moll, H., Denecke, M. A., Geipel, G. Bernhard, G., Nitsche, H., Allen, P. G., Buchner, J. J., Kaltsoyannis, N., Edlestein, N. M. and Shuh, D. K. (1996). Characterization of hydrous uranyl silicate by EXAFS. Radiochim. Acta 74, 219-223.

${ }^{63}$ Teo, B. K. (1986). EXAFS: Basic Principles and Data Analysis. Inorganic Chemistry Concepts, Vol. 9, Springer-Verlag. Berlin.

${ }^{64}$ Griffiths, T. R. and Volkovich, V. A. (1999). A review of the high temperature oxidation of uranium oxides in molten salts and in the solids state to form alkali metal uranates, and their composition properties. J. Nucl. Mater. 274, 229-251. 
${ }^{65}$ Farges, F., Ponader C. W., Calas G. and Brown, G. E. Jr. (1992). Structural environments of incompatible elements in silicate glass/melt systems: II. $\mathrm{U}^{\mathrm{IV}}, \mathrm{U}^{\mathrm{V}}$, and $\mathrm{U}^{\mathrm{VI}}$. Geochim. Cosmochim. Acta 56, 4205-4220.

${ }^{66}$ Shannon, R. D. (1976). Revised effective ionic radii and systemic studies of interatomic distances in halides and chalcogenides. Acta Cryst. A32, 751-767.

${ }^{67}$ Duff, M. C., Coughlin, J. U. and Hunter, D. B. (2002). Uranium co-precipitation with Fe oxide minerals. Geochim. Cosmochim. Acta 66, 3533-3547.

${ }^{68}$ A discussion of the uranate structures and misclassifications is an important topic but such discussion is not warranted in this report given the results that will be discussed.

${ }^{69}$ Hobbs, D. T. and Edwards, T. B. (1993). "Solubility of Uranium in Alkaline Salt Solutions," WSRCTR-93-00454. May 1993.

${ }^{70}$ Hobbs, D. T., Edwards, T. B. and Fleischman, S. D. (1993). "Solubility of Plutonium and Uranium in Alkaline Salt Solutions,” WSRC-TR-93-00056. February 1993.

${ }^{71}$ Because of its similar size, Al may be present instead of Si, but to date, no crystalline uranyl aluminate solids have been identified, suggesting that bonding to $\mathrm{Si}$ is much more likely than to Al.

${ }^{72}$ Smith, D. K. (1984). Uranium mineralogy. In Uranium Geochemistry, Mineralogy, Exploration and Resources (ed. B. DeVivo, F. Ippolito, G. Capaldi and P. R. Simpson). pp. 43-88. Institute of Mining and Metallurgy.

${ }^{73}$ Pérez, I., Casas, I., Martín, M. and Bruno, J. (2000). The thermodynamics and kinetics of uranophane dissolution in bicarbonate test solutions. Geochim. Cosmochim. Acta 64, 603-608.

${ }^{74}$ Perez, I., Casas, I., Torrero, M., Cera, E., Duro, L., and Bruno, J. (1997). Dissolution Studies of Soddyite as a Long-Term Analogue of the Oxidative Alteration of the Spent Nuclear Fuel Matrix, Scientific Basis for Nuclear Waste Management XXI. MRS Symposium Proceedings, 465, 565-572. Pittsburgh, PA: Material Research Society.

${ }^{75}$ Mason, C.F.V., Duff, M. C., Musgrave, J. A. and Runde, W. (1996). "Interim Report on Remedial Actions for Yuma Catch Box Sand," Los Alamos National Lab., Los Alamos, NM, LA-UR 96-3485 Oct. 1996. 